$$
\text { DOEIERIIZnS2..TI }
$$

\title{
Generation of Radiation by Intense Plasma and Electromagnetic Undulators
}

\section{FINAL TECHNICAL REPORT}

Submitted by:

THE REGENTS OF THE UNIVERSITY OF CALIFORNIA

UNIVERSITY OF CALIFORNIA, LOS ANGELES

SCHOOL OF ENGINEERING AND APPLIED SCIENCE

LOS ANGELES

PRINCIPAL INVESTIGATOR:

C. Joshi, Professor

Electrical Engineering Department

(310) 825-7279

Period of performance: July 1, 1987-September 30, 1990

submitted to:

Dr. Walter M. Polansky

U.S. DEPARTMENT OF ENERGY

Office of Basic Energy Research

for

Grant Number DE-FG03-87-ER13752

OCTOBER 1991 


\section{DISCLAIMER}

This report was prepared as an account of work sponsored by an agency of the United States Government. Neither the Unitcd States Government nor any agency thereof, nor any of their employees, makes any warranty, express or implied, or assumes any legal liability or responsibility for the accuracy, completeness, or usefulness of any information, apparatus, product, or process disclosed, or represents that its use would not infringe privately owned rights. Reference herein to any specific commercial product, process, or service by trade name, trademark, manufacturer, or otherwise does not necessarily constitute or imply its endorsement, recommendation, or favoring by the United States Government or any agency thereof. The views and opinions of authors expressed herein do not necessarily state or reflect those of the United States Go:'ernment or any agency thereof. 


\section{Introduction}

The following pages contain the details of the work carried out under the DOE grant number DE-FG03-87-ER13752.

\section{Studies of Classical Radiation Emission From Plasma Wave Undulators.} This report comprises the work that was performed during the third year of this grant, July 1, 1989 through September 30, 1990. This manuscript will be submitted to the Laser and Particle Beams journal for publication.

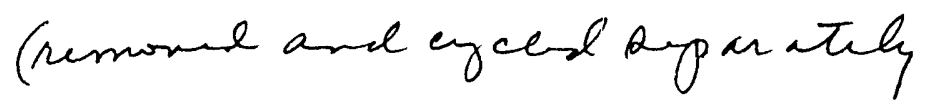

II. Second Year Progress Report. This progress report was submitted to the DOE previously. The report summarizes the work performed during the second year of this grant, July 1, 1988 through June 30, 1989. (renome ard eyeed pegaratily)

III. First Year Progress Report. This report summarizes the work performed during the first year of this grant, July 1, 1987 through June 30, 19898. (Mrmoned and

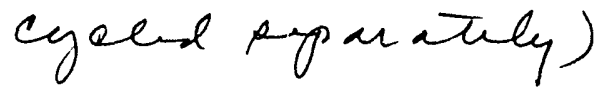

IV. Evaluation of Millimeter and Microwave Source Capabilities. This is the final report from Spectra Technology. It summarizes the work resulting from- the UCLA subcontract under this grant to Spectra Technology.

V. Publications. This is a listing of the publications resulting from work that was supported by this DOE grant. The actual publications are attached in the appendix.

VI. Appendix. (remoned ard cychel pegarialy) 
I. Studies of Classical Radiation Emission From Plasma Wave Undulators. 
(removed and cyead Regaratub) -

Studies of Classical Radiation Emission From

Plasma Wave Undulators

R.L. Williams, C.E. Clayton and C. Joshi

Department of Electrical Engineering

University of California, Los Angeles, Los Angeles, CA 90024

T. Katsouleas

Department of Electrical Engineering-Electrophysics

University of Southern California, Los Angeles, CA 90089-0484

ABSTRACT

We examine the characteristics of the classical radiation emission resulting from the interaction of a relativistic electron beam that propagates perpendicularly through a large amplitude relativistic plasma wave. Such a study is useful for evaluating the feasibility of using relativistic plasma waves as extremely short wavelength undulators for generating short wavelength radiation. The electron trajectories in a plasma wave undulator and in an ac FEL undulator are obtained using perturbation techniques. The spontaneous radiation frequency spectrum and angular distribution emitted by a single electron oscillating in these two undulators are then calculated. The radiation gain of a copropagating electromagnetic wave is calculated. The approximate analytic results for the trajectories, spontaneous radiation and gain are compared with 3-D simulation results. The characteristics of the plasma wave undulator aie compared with the ac FEL undulator and linearly polarized magnetic undulator.

Prepared for submittal to Laser and Particle Beams 
IV. Evaluation of Millimeter and Microwave Source Capabilities. 


\title{
EVALUATION OF MILLIMETER AND
} MICROWAVE SOURCE CAPABILITIES

\section{Final Technical Report}

\author{
Submitted to \\ University of Califomia, Los Angeles \\ 7731 Boelter Hall \\ 405 Hilgard Avenue \\ Los Angeles, California 90024
}

by

Spectra Technology, Inc.

2755 Northup Wayl

Bellevue, Washington 98004

30 SEPTEMBER 1990 
Table of Contents

SECTION

PAGE

1

Introduction

1-1

2

Conventional Source Summary

2-1

2.1 Solid State Source

2-1

2.2 Vacuum Electronic Sources

2-3

3

Applications Introduction

3-1

3.1 Crafted Wave Impulse Applications

3.2 Other Applications

3-2

3-5

4

Solid State Specifics

4-1

4.1 Introduction

4-1

4.2 IMPATT Diodes

4.3 Gunn Diodes

4-5

4.4 TUNNEL Diodes

4.5 TUNNETT Diodes

4-20

4.6 BARRITT Diodes

4-22

4.7 TRAPATT Diodes

4-22

4.8 MITATT Diodes

4.9 DOVETT Diodes

4-23

4-23

4-23

4.10 FET's and Bipolar Transistors

4-24

4.11 Harmonic Generators

4-24

4.12 Power Combining

4.13 Manufacturers [ref 46]

4-25

4-25

5

Vacuum Electronics Specifics

5-1

5.1 Conventional Slow Wave Devices

5-1

5.2 Wide Aperture Slow Wave Devices

$5-3$

5.3 Fast Wave Devices

$5-5$

6 Summary and conclusions

6-1

References

R-1 


\section{LIST OF FIGURES}

2-1 Source Power Capability Summary 2-2

$2-2$

$2-3$

History of Average Power

$2-4$

Average Power Output vs. Frequency for Backward $\quad 2-5$

3-1 Frozen Wave Generator Concept

3-3

3-2 Generation of Crafted mm Wave using Plasma Upshift 3-6

with Shaped UV Ionizing Pulse

3-3 Atmospheric Absorption of $\mathrm{mm}$ Waves $3-8$

4-1 Comparison of conventional Millimeter Wave Sources 4-2

4-2 Comparison of Solid State Sources

4-3 Qualitative Comparison of solid State Source 4-4

4-4 Typical Oscillator Configuration with solid State 4-6

4-5 Typical Amplifier configuration Utilizing Solid State 4.7

4-6 Schematic Diagram of Two Manifestations of IMPATT 4.9

4.7 Current Technology in Pulsed IMPATT Power 4-10

Capability Based on Data from Hughes, Plessey

4-8 CW IMPATT Power Capability Based on Data from

4-11

Hughes, Plessey, and Raytheon

4.9 Efficiency of Selected Solid State Devices 4.12

4-10 Typical Packages for IMPATT Diodes 4-15

4-11 Simplified Schematic Diagram of Energy Level in a 4-16

4-12 Comparison of Small-Signal Noise Measures for 4-17

Various Semiconductor Microwave Devices in the

Frequency Range 8 to $12 \mathrm{GHz}$

4-13 Output Microwave Power vs. Frequency for Pulsed and $4-20$

4-14 Cw-Operated GaAs and InP TEDS

4-15 Power Combining Using Rectangular Resonator 4-26

4-16 Power Combining Configurations 4-27

5-1 Examples of Slow Wave Structures

5-2 Overmoded, Wide Aperture Slow Wave Structures 5-4

5-3 Gyrotron 5 5-7

5-4 FEL Woncept $5-9$ 


\section{LIST OF TABLES}

TABLE

PAGE

2-1 Points of Interest in Development of Tunable,

$2-8$

High Power, Microwave/mm Sources

3-1 Dominant Uses of the Millimeter and Microwave

3-7 Spectrum

3-2 Applications Requiring Bandwidth, Tunability, and

3.9 Moderate Power 


\section{LIST OF FIGURES}

FIGURE

PAGE

2-1 Source Power Capability Summary 2-2

2-2 History of Average Power 2-4

2-3 Average Power Output vs. Frequency for Backward 2-5

Wave Oscillator

3-1 Frozen Wave Generator Concept 3-3

3-2 Generation of Crafted mm Wave using Plasma Upshift 3-6

3-3 Atmospheric Absorption of $\mathbf{m m}$ Waves

4-1 Comparison of conventional Millimeter Wave Sources 4-2

4-2 Comparison of Solid State Sources 4-3

4-3 Qualitative Comparison of solid State Source 4-4

4.4 Typical Oscillator Configuration with solid State 4-6

4-5 Typical Amplifier configuration Utilizing Solid State 4-7

4-6 Schematic Diagram of Two Manifestations of IMPATT 4-9

4-7 Current Technology in Pulsed IMPATT Power 4-10

Capability Based on Data from Hughes, Plessey

4-8 CW IMPATT Power Capability Based on Data from 4-11

4-9 Efficiency of Selected Solid State Devices $4-12$

4-10 Typical Packages for IMPATT Diodes 4-15

4-11 Simplified Schematic Diagram of Energy Level in a 4-16

4-12 Comparison of Small-Signal Noise Measures for 4-17

Various Semiconductor Microwave Devices in the

Frequency Range 8 to $12 \mathrm{GHz}$

4-13 Output Microwave Power vs. Frequency for Pulsed and 4-20

4-14 Tunnel Diode Characteristics

4-15 Power Combining Using Rectangular Resonator 4-26

4-16 Power Combining Configurations 4-27

5-1 Examples of Slow Wave Structures 5-2

5-2 Overmoded, Wide Aperture Slow Wave Structures 5-4

5-3 Gyrotron 5-7

5-4 FEL Concept 


\section{LIST OF TABLES}

TABLE

PAGE

2-1 Points of Interest in Development of Tunable,

$2-8$ High Power, Microwave/mm Sources

3-1 Dominant Uses of the Millimeter and Microwave

3-7 Spectrum

3-2 Applications Requiring Bandwidth, Tunability, and

$3-9$ Moderate Power 


\section{SECTION 1 \\ INTRODUCTION}

This report h\% been prepared to provide perspective concerning the potential and desirability of developing new microwave and millimeter radiation sources. Specifically, it compares existing sources. along with several others under development, to a new concept of up-conversion by mer.zs of a modulated plasma [ref 1,2$]$. The latter approach may lead to a versatile, compact sources with extreme tunability, perhaps form 30 to $600 \mathrm{GHz}$ in a single device, compared to roughly factor of two maximum range from more conventional sources. Frequency upshifting may be a viable generation mechanism for a variety of laboratory, commercial, and military applications.

The report draws a comparison between the fundamentals of the existing sources and the plasma upshifted source. For the purposes of the discussion, the potential parameters assumed for the shifted source are given in Table 1-1. The frequency upshifting occurs when a plasma is rapidly created in a volume which initially contains a propagating EM wave in an unionized gas. The initial wave is not of sufficient intensity of frequency to ionize, and the ion population is created by a second, higher frequency wave. If the plasma is overdense for the initial wave, then this wave is upshifted to approximately the critical frequency. The wavelength shift is adjustable through variation of the plasma density. In the first demonstration, by Joshi[ref 2], a wave of $33 \mathrm{GHz}$ was shifted approximately $35 \mathrm{GHz}$ with 10 percent efficiency and frequency components to $77 \mathrm{GHz}$ were observed.

\section{rable 1-1 \\ ASSUMED PARAMETERS FOR PLASMA UPSHIFTED MIIDMIETER WAVE SOURCE}

Frequency Range

Intrinsic Conversion Efficiency of $30 \mathrm{GHz}$ Input

Peak Power

Maximum Repetition Rate
$30-600 \mathrm{GHz}$

$50 \%$ at $30 \mathrm{GHz}$

$0.1 \%$ at $600 \mathrm{GHz}$

$100 \mathrm{~kW}$ at $30 \mathrm{GHz}$

$10 \mathrm{~W}$ at $600 \mathrm{GHz}$

$10 \mathrm{kHz}$ 
One interesting opportunity for such a source is the ability to craft waveforms through rapid modulation of the plasma frequency, leading to shifts controlled nearly on a period by period basis. Similarly, a highly chirped wave can be formed with subsequent generation of an ultra short pulse in some dispersive medium. Compressed pulses, including those with additional modulation within their short length, are attracting much attention within the radar community, where the potential benefits [ref 3] of such waveform crafting include the detection stealth targets. That prospect is generating vibrant discussion with respoct to the Northrup B2 stealth bomber project [ref 4].

The report is organized as follows. Section 2 contains a short summary of the capability of conventional sources, including discussion of the principles which limit tunability. The assumed capabilities of the plasma upshifted source are compared to both solid state and vacuum electronic sources. Section 3 summarizes current applications for microwave and millimeter waves with emphasis on those requiring tunability. Sections 4 and 5 contain additional information on capabilities and limitations of conventional sources that is not in the source summary of Section 2.0. 


\title{
SECTION 2 \\ CONVENTIONAL SOURCE SUMMARY
}

\begin{abstract}
A summary of the available sources is shown in Figure 2-1, which also includes the assumed parameters for plasma upshift source as previously given. The existing sources fall into categories of solid state, vacuum electronic, and quantum devices. As seen in the figure, solid state devices lag the vacuum electronic (tube) devices by roughly five onders of magnitude in power, and are not in use above several hundred $\mathrm{GHz}$. The slow-wave tubes involve structure sizes that are generally less than a wavelength in cross section, and therefore do not scale simultaneously to high frequency and high power. This constraint is partially avoided with the gyrotron, which allows a waveguide width of up to several waves. In the free electron laser (FEL) this constraint of waveguide width is absent entirely, and the active cross section is essentially unlimited. The advantages of this with respect to power and frequency are apparent from the figure in the Induction Linac FEL curve. The reader may know that the FEL wiggler bore size is often narrowed to make a large magnetic field possible, which would appear to contradict the statement above. However, the wiggler can, in priaciple, be removed and replaced with a counter propagating EM wave of low frequency.
\end{abstract}

\subsection{SOLID STATE SOURCES}

The solid state devices dominate at low power, and at this time the only opportunity for these devices to move to higher power is by beam combination. There are three broad types of solid state millimeter devices, two terminal devices, three terminal devices, and harmonic generators or frequency multipliers. They cover a frequency range to about $200 \mathrm{GHz}$ at peak powers under a hundred watts as shown in Figure 2-1. At much lower powers they can be extremely broadband, leading to versatile, general purpose devices such as spectrum analyzers.

The two terminal devices (e.g. IMPATT and Gunn) operate on a negative differential resistivity, involving a time lag between current and voltage, whose frequency characteristics are dependent on the specific construction and use of the device. The devices are generally used in conjunction with a resonant cavity as a means of frequency control. Tixey can be tuned electronically by veractor diodes 


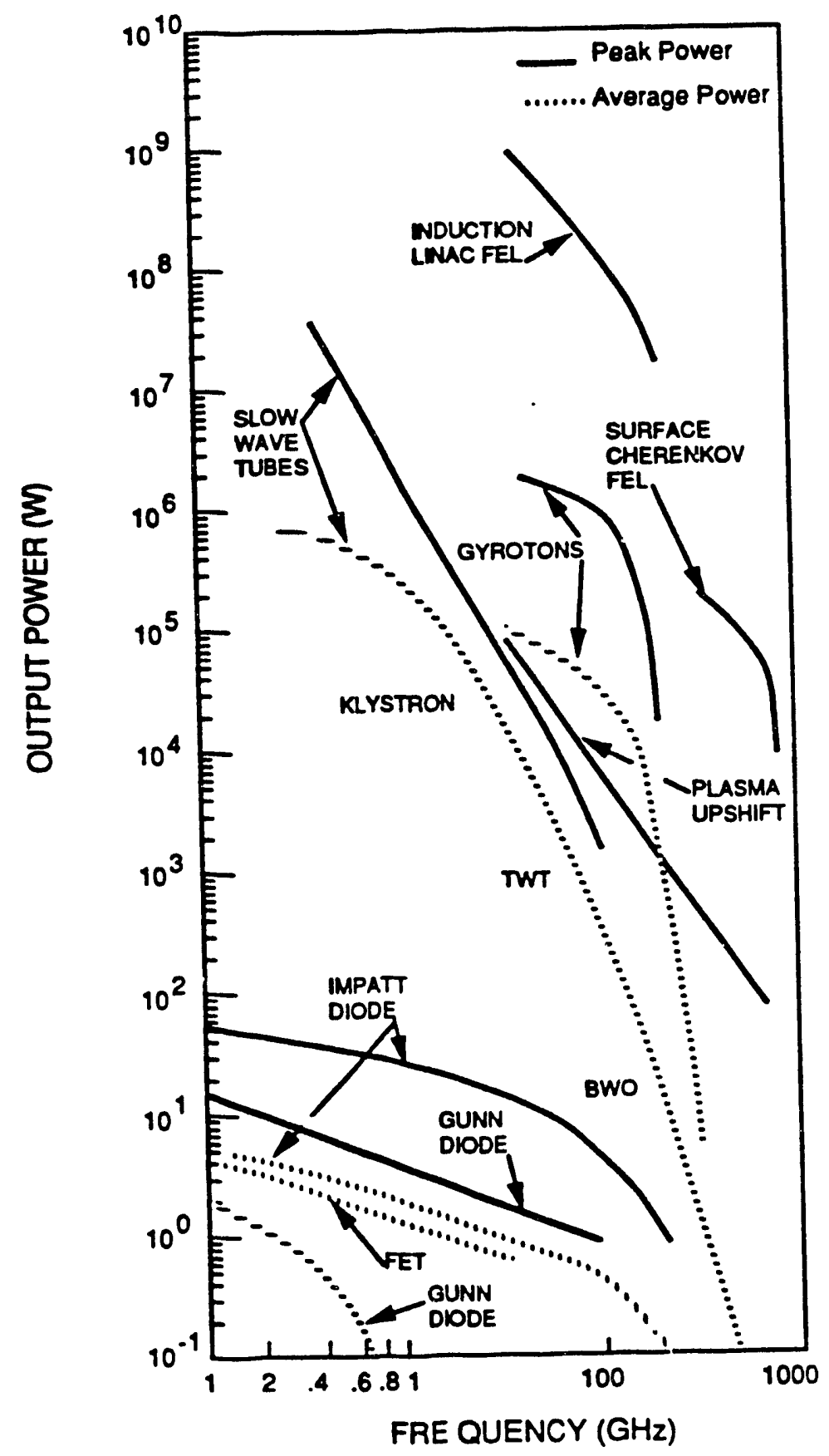

$\infty 010099$

Figure 2-1. Source Power Capability Summary. Adapted in part from References 5-8. 
through a typical range of about 5 percent, and mechanically tuned with the resonant cavity through a range of 20 to 30 percent. The tuning range can be dramatically larger if power and efficiency are unimportant.

The common three terminal devices are FETs and bipolar transistors. The FET's operate to $100 \mathrm{GHz}$, but can produce significant power only to about $35 \mathrm{GHz}$, since the dimensions of the gate at higher frequencies are becoming less than a micron. Bipolar transistors are in principle capable of operation at several hundred $\mathrm{GH}_{\mathbf{z}}$, but they have received relatively little attention at high frequencies because of low gain, low efficiencies, poor noise figures, and other disadvantages compared to the FET.

The harmonic generators are generally two port devices used in cross guide configurations in which input power enters one port and frequency multiplied power exits the other. These multipliers are capable of $100 \mathrm{~mW}$ power levels up to $200 \mathrm{GHz}$ and some power has been obtained to $600 \mathrm{GHz}$. However, careful tuning is required to suppress unwanted harmonics. This greatly restricts their tuning range and therefore they not considered further.

\subsection{VACUUM ELECTRONIC SOURCES}

The vacuum electronic devices produce orders of magnitude higher powers than the solid state devices, and in some cases operate at higher frequencies. A simple summary of the vacuum electronic devices including their evolution over the years is given in Figure 2-2. At lower frequencies the most used and oldest sources are the magnetrons and klystrons. These devices contain critical mechanical components for frequency control and they are in general not tunable over more than several percent.

One of the first exceptions to this tunability constraint was the backward wave oscillator (BWO) which provided not only higher frequency operation than the klystrons and magnetrons, but is routinely used over the roughly $40 \%$ width of various waveguide bands. The present highest frequency demonstration is slightly over $1200 \mathrm{GHz}$ [ref 22]. With the large tunability, the BWO is rapidly becoming the workhorse of the millimeter frequency regime. As such it deserves special attention in the context of this is report. Figure 2-3 shows the available average output power in the 30 to $1000 \mathrm{GHz}$ range. This limit is set by the small tube cross section, which is well under one wavelength. The potential tunability throughout this range is 


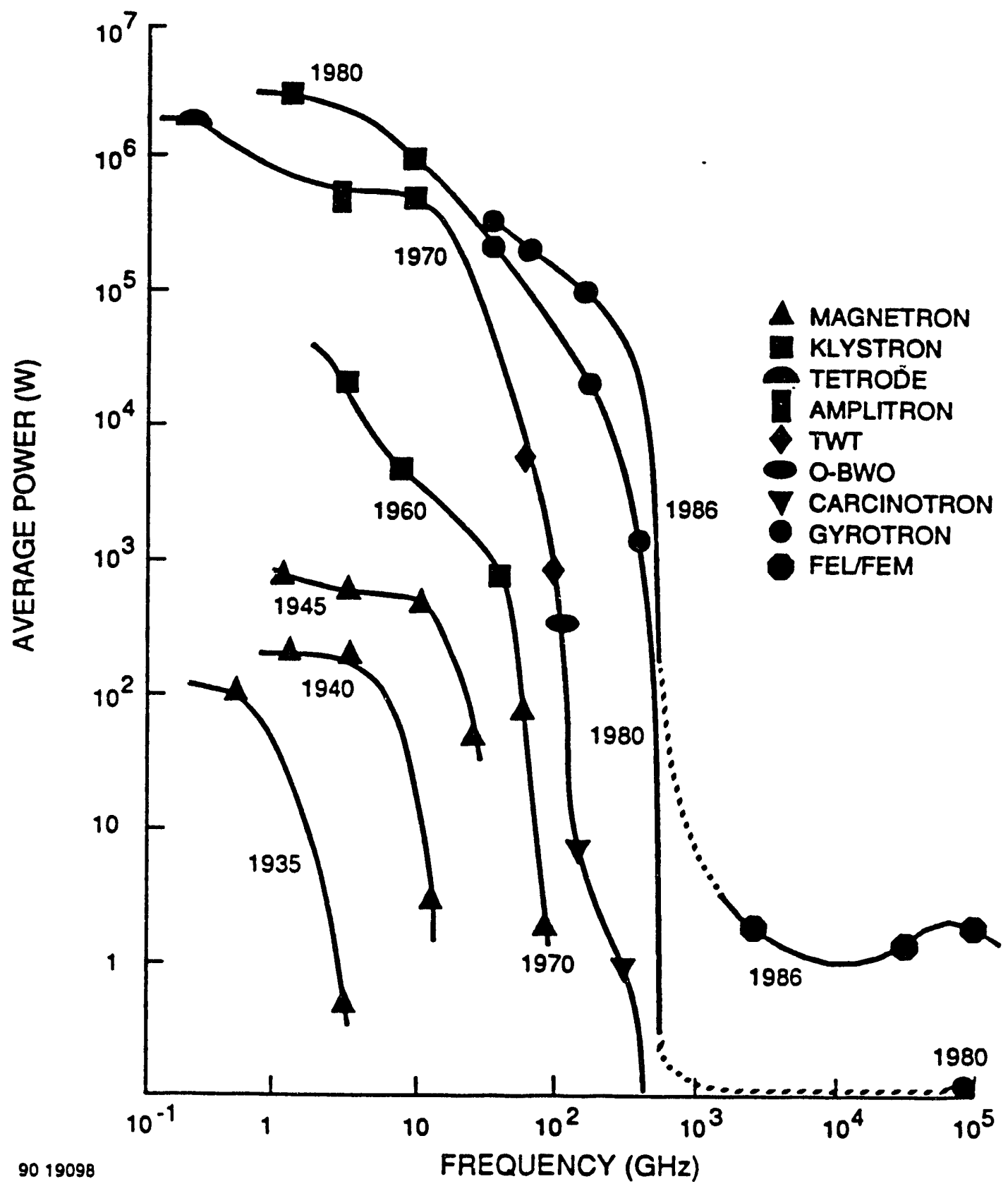

Figure 2-2. History of Average Power. Adapted from Reference 5. 


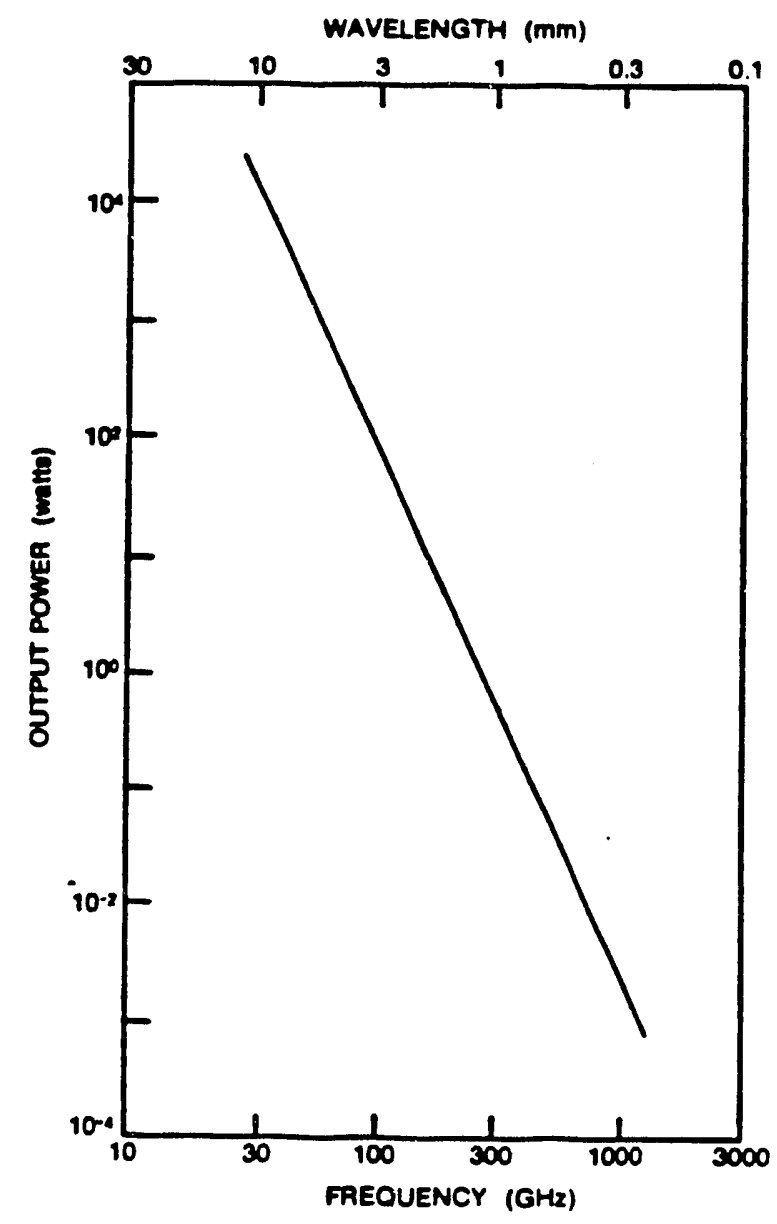

Figure 2-3. Average Power Output vs. Frequency for Backward Wave Oscillator. Tuning range for single device is one octave. Taken from Reference 6 . 
approximately one octave. Pulsed powers [ref 23] have been produced in the 35 to $125 \mathrm{GHz}$ region with approximately four orders of magnitude higher power than shown.

Interestingly, the evolution to shorter wavelength and higher power of the vacuum devices is also leading to larger tunability. The use of resonant mechanical cavities in the slow wave devices limits the device size and hence heat removal. capability, and of course it also limits the tunability. Partial relief from the cavity size constraint came with development of the gyrotron, which does not require a slow wave structure and allows a tube cross section of several wavelengths. However, the tunability is still lacking since the mode geometry must be carefully controlled to force the desired interaction. This is especially critical if radiation at harmonics of the cyclotron frequency or high mode numbers is desired. The requirement of waveguide wall proximity will likely limit the ultimate pulsed power limit of the gyrotron to roughly $100 \mathrm{~mW}$ at $100 \mathrm{GHz}$, and the ultimate frequency maximum is likely less than $300 \mathrm{GHz}$.

Larger cross sections (than with the BWO) are also possible in slow wave structures. Examples are the Ledatron and the related Orotron [Ref 6,14], neither in widespread use. In these devices a grating and facing mirror form a resonator which can have many waves of separation. Interaction with the electron still requires proximity to the metal surface, however. The devices have the potential of roughly an octave tuning range by using both mechanical and electrical adjustment.

A related broad band device is the Cherenkov laser, which uses a hollow dielectric waveguide as a slow wave structure. A series of devices have been built and tested by Walsh [ref 7, 20,21] at $4 \mathrm{~mm}$ wavelength and shorter, and one single device has achieved lasing from 375 to 1000 microns. These differ from other slow wave devices discussed above in that no periodic structure is employed. Accordingly the ultimate tuning range may be extended because the usual structure of pass and stop bands associated with the periodic structures is absent. Capabilities of the Walsh devices are shown in Figure 2-1 as Surface Cherenkov FELs. As with other other slow wave devices, proximity to a wall with boundary parallel the the e-beam propagation is still required.

If no slow wave structure is needed for phase matching, as is the case with the gyrotron, the necessary mode selection can, in principle, be achieved without surfaces close to the e-beam by simply using the end mirrors in an optical cavity geometry. The 
mode is controlled by beam truncation. Removal of the walls removes the attendant power limitations if the optical beam is allowed to expand as needed before striking the end mirrors. Such a scheme would be possible with the gyrotron mechanism and appears feasible in initial studies [REF 9].

With the first operation of the FEL a fastwave resonance mechanism has been demonstrated which allows electron-photon energy exchange both at any wavelength (in principle) and without wall proximity to the e-beam. The ability to develop large powers with the FEL shows clearly in Figure 2-1. The FEL has been demonstrated from centimeter [ref 15] to ultraviolet [ref 16] wavelengths and even has proponents for eventual $x$-ray facilities.

Unfortunately, there is a significant penalty associated with the phase matching as embodied in the FEL. It suffers from from property that the electron beam motion is nearly orthogonal to the optical E-field, causing weak coupling between the electrons and photons. The result is poor gain, poor efficiency, or both. In practical systems, the FEL oscillator is gain starved as one approaches the IR spectrum from the millimeter direction, and at longer wavelengths it is not generally competitive with devices in which the e-beam motion is more aligned with the photon E-field, e.g. the gyrotron. Virtually none of the slow wave devices suffer from this difficulty, but of course they are inherently not scalable to large power or short wavelength.

Practical considerations have lead to an FEL which is large, expensive, and difficult to maintain, at least for the present. Clearly the FEL has yet to be fully developed, and in the meantime it carries great potential while being extremely specialized. To date the largest tuning range for a single device is approximately a factor of four, from 9 to 35 micron at Los Alamos [ref 17]. Similar fractional spreads have since been obtained in other experiments spanning from the UV to $1 \mathrm{~cm}$ wavelengths [refs $11,15,16$. But with its complexity it is doubtful that the FEL will ever be a popular source in the millimeter range except for high power applications.

Table 2-1 highlights several of the key device characteristics that have occurred in the progression from the early microwave devices with extremely linited tuning range to the more recent shorter wavelength devices with much larger tuning ranges. In this table the slow and fast wave designations refer to whether or not the phase velocity of the EM wave is slowed to the velocity of the electrons. The plasma upshift source is most closely related to last category listed, as basic mechanism is completely broadband and wall contact is not a fundamental requirement. 


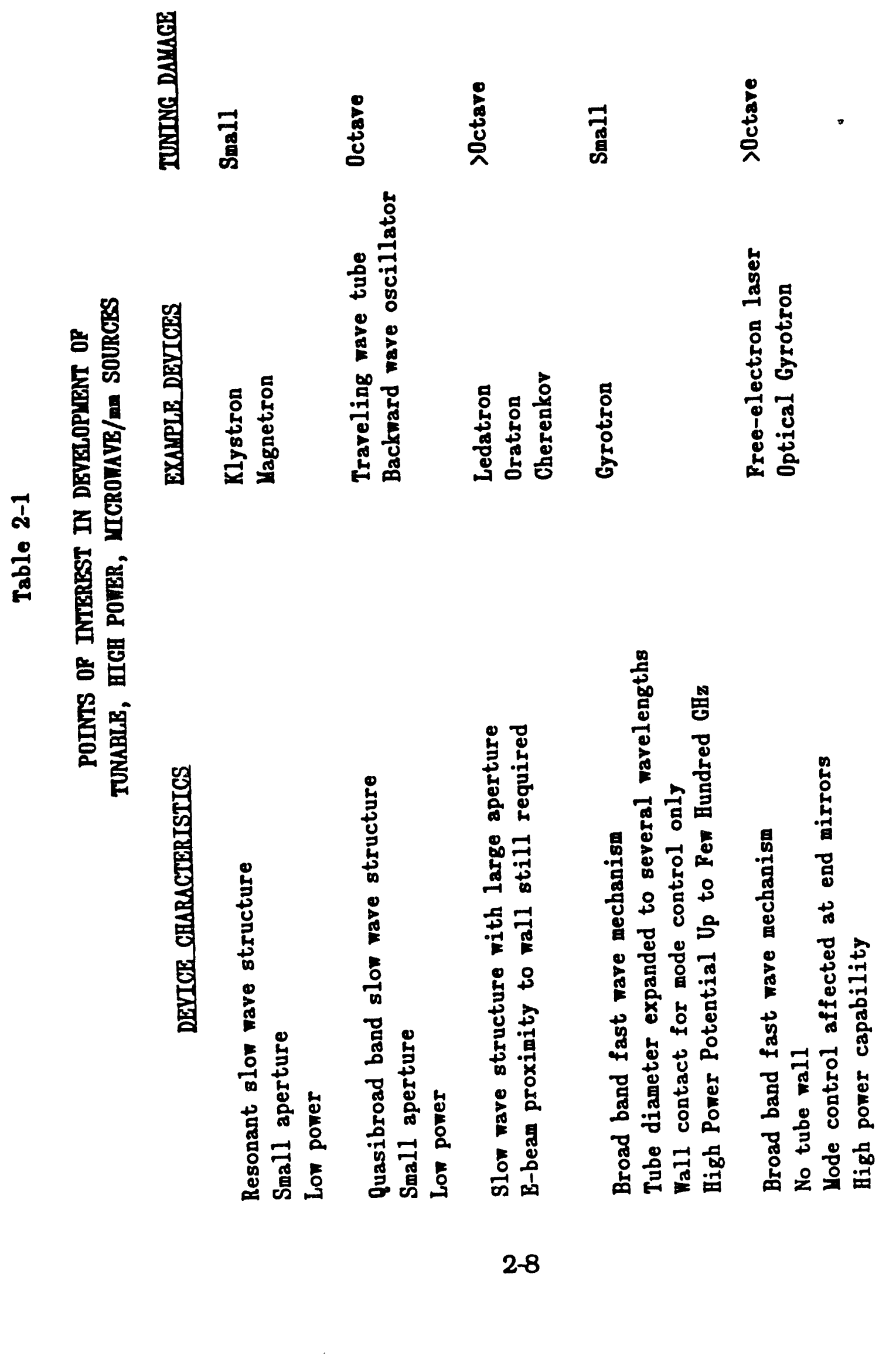




\section{SECTION 3 \\ APPLICATIONS INTRODUCTION}

The millimeter wave region, defined approximately as $30-300 \mathrm{GHz}$, is not seeing the level of research and development that might have been expected some years ago. With the maturing of the microwave field in the 1960's, there had been a general expectation that the millimeter wave technology would maintain the development cycle that that had seen the explosive growth of microwave radar and communications systems.

Among the expected advantages of the millimeter waves as compared to that for microwaves are size, cost, and reliability of components, greater bandwidth, smaller beam sizes and improved angular resolution. The increased interaction with atmospheric constituents was expected to lead to a host of remote sensing applications.

With the exception of new solid state millimeter wave sources, the widescale development of millimeter component technology has yet to occur. Fundamental components such as waveguides, directional couplers, etc are still generally based on 1950's and 1960's designs, and by microwave standards the performance of many of these components is poor at the higher frequencies. There is no volume production of integrated components (e.g. source, receiver, and signal analysis) that could be expected based on the success of solid state sources.

For the most part the lack of development can be related to low DOD development funds in these areas, and this in turn is a result of, in some key applications, bypassing the millimeter region and proceeding directly to the $\mathbb{R}$. An excellent example is the $\mathbb{R}$ missile guidance (e.g. ground- to-air seekers) now being vigorously pursued. The compact size, applicable optical techniques, and integrated production techniques available for these components makes them extremely attractive.

The all-weather capability generally required for DOD applications cannot, however, be achieved with IR systems. For example, the now popular mid-IR seeker systems (2-5 microns) will not function properly in clouds. The millimeter systems can operate in clouds but not rain, with the result that the highest frequency for all-weather capability is the microwave spectrum up to about $20 \mathrm{GHz}$. This explains, in part, why the development effort has to some extent bypassed the millimeter wave region. 


\subsection{CRAFTED WAVE IMPULSE APPLICATIONS}

There is a new trend developing in military and other applications in the microwave to IR range that is particularly relevant to the context of this report. It falls under the general heading of complex waveforms, including simultaneous or sequential use of discrete frequencies, waveforms heavily modulated in frequency and amplitude, and even ultra wideband emission with bandwidth approaching unity. Such waves can be crafted with the plasma upshift technique, in principle, and as one proceeds to higher frequencies from microwaves, plasma upshifting may offer waveform control that is not possible through other means.

The ultra wide bandwidth (UWB) field is entirely new, and the applications are still quite speculative. It is generating considerable interest as it applies to radar in general [ref 3], and detection of stealth systems such as the B2 bomber in particular [ref 4]. The wide bandwidth may be associated with a large frequency chirp in a long wave train, an unchirped wave only a few cycles long, or a shor wave of several cycles which is also heavily phase and amplitude modulated. Among the advantages claimed for UWB radar over conventional radar are

1) Higher range resolution

2) Improved target identification capability

3) Detection of low observable (stealth) targets

4) Improved penetration (earth, foliage, rain, walls) capability

5) Rejection of clutter through range gating

6) Reduced sensitivity to electronic countermeasures

At frequencies up to several $\mathrm{GHz}$ it possible to craf $\iota$ arbitrary waveforms with a class a techniques which can be called Frozen Wave Generators. The concept, as depicted in Figure 3-1, begins with a transmission is segmented into short sections whose center conductors are electrically isolated. These sections may be charged on a long timescale to various different voltages, after which the individual sections of the transmission are quickly connected. A wave begins propagating within the line in both directions at the time of connection. The complexity of the wave, or equivalently the 


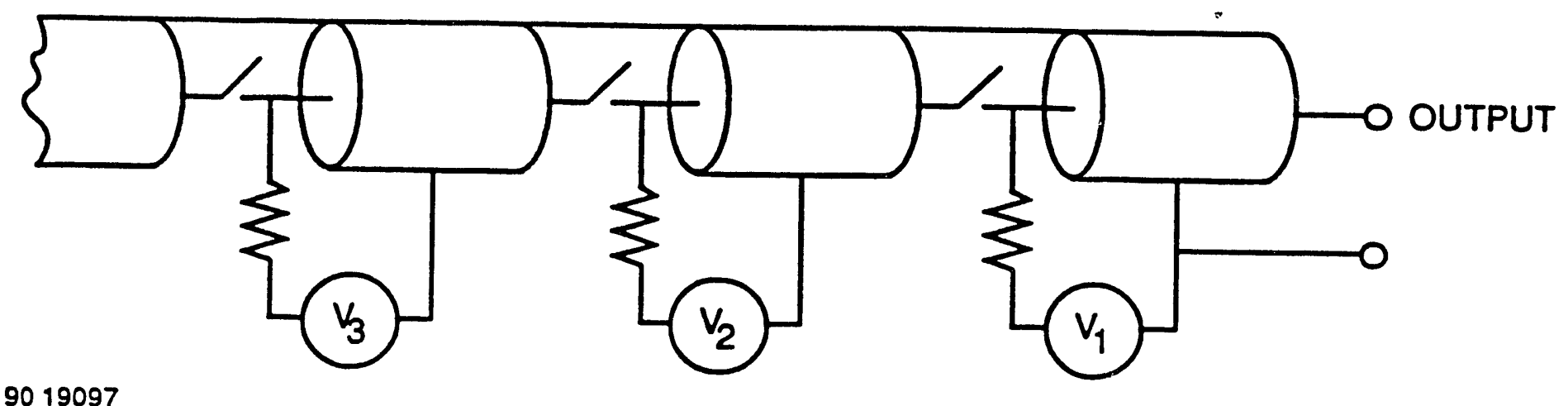

9019097

Figure 3-1. Frozen Wave Generator Concept. Running waves of arbitrary shape are launched if switches are closed simultaneously after charging of individual transmission line sections to different, predetermined potentials. 
highest frequency components, is limited only by the length of the individual segments and the switching speed. Unidirectional transmission is possible if the currents as well as potentials of individual sections are pre-established.

The switch technology is obviously a key element. A bulk avalanche semiconductor (BASS) switch is in development [ref 18] that can be triggered with power lasers and which then continues to conduct until the current flow stops. As the desired frequency increases the components must become smaller and an increasing fraction of the transmission line is occupied by the switches. The switch size then becomes the frequency limiting element, even if the switch speed is still appropriate. This likely limits the BASS switch technique to UWB radar at frequencies below $10 \mathrm{GHz}$.

The plasma upshift technique may provide a means to create crafted waves at still higher frequencies. One concept for modulated shifting with the plasma is based on modulation of the plasma frequency by modulation of the UV ionizing intensity. This scheme is best applicable to the cases where it is desired to create a millimeter wavetrain with a monotonic upshift in frequency, i.e. a wavetrain with the lowest frequency components at the front and progressively higher frequency components as one moves through the train.

The plasma density, and therefore the frequency of the output wave, is temporally modulated according to the intensity of the UV ionizing wave. Under conditions for which the electron-ion recombination time is longer than the length of the incoming UV pulse, say 10 periods of the millimeter wave, and the ionized species is not saturated, then the plasma created near the window has an increasing density for the period of interest. The millimeter wave emerging from the window will be at approximately the plasma frequency. The plasma density is simply related to the time integrated optical flux, so that

$$
\omega_{\mathrm{mm} \text { wave }} \sim \omega_{\mathrm{p}} \sim\left[\int \text { Intensityuv(t)dt }\right]^{1 / 2}
$$

The intensity temporal profile can be controlled to generate a desired temporal history of the output wave. 
An implementation schematic diagram is shown in Figure 3-2. This scheme is applicable to control of short wave trains, like those of inuerest to the radar research community. The input UV ionizing pulse is shown on the right hand side. This pulse is assumed to be have a predictable temporal profile and would have a pulse length of roughly 10 periods of the millimeter wave, perhaps 100 ps in a $3 \mathrm{~cm}$ pulse length. This pulse is in a plane wave that uniformly illuminates the Bundled-Rod Pulse Shaper. The rods are simple delay lines according to their length. The distribution of lengths controls the temporal power profile of the transmitted UV pulse. The rods locations in the bundle are not critical, and if there is a large number as well as random location within the bundle, the transmitted beam will be roughly homogenized in the transverse dimension. Without homogenization, spatial variation in the density of the upshifting plasma would occur.

Transverse homogenization is further assured by the additional optical elements shown. This arrangement homogenizes without significant loss of photons, and obviates the need for randomly distributing the delay lines. It is related to that devised by Phalen et al [ref 12]. The Image Separation Lens Array separates the wave into a number of discrete point sources. Light from those point sources is transformed to the far field and combined spatially by the Far-Field Homogenizing Lens. The combination occurs in the spatial plane labeled Object Plane. Light from each point source is mapped evenly within the high intensity area of the crossing. This plane is relayed to the Image Plane near the front of the plasma upshift cell. Reasonable uniformity of illumination is required over a plasma volume that contains the length of the wavetrain to be crafted. If this condition is not met, reflections internal the plasma will complicate the output waveform. Output millimeter radiation emerges from the same end of the cell the UV enters, and is separated by a dichroic splitter. Fine wire mesh is suitable for the millimeter frequencies.

\subsection{OTHER APPLICATIONS}

A very concise summary of current millimeter and microwave applications is given in Table 3-1. The area of both largest dollar expenditure and largest application growth is the military. Here the traditional applications have been sadar and 


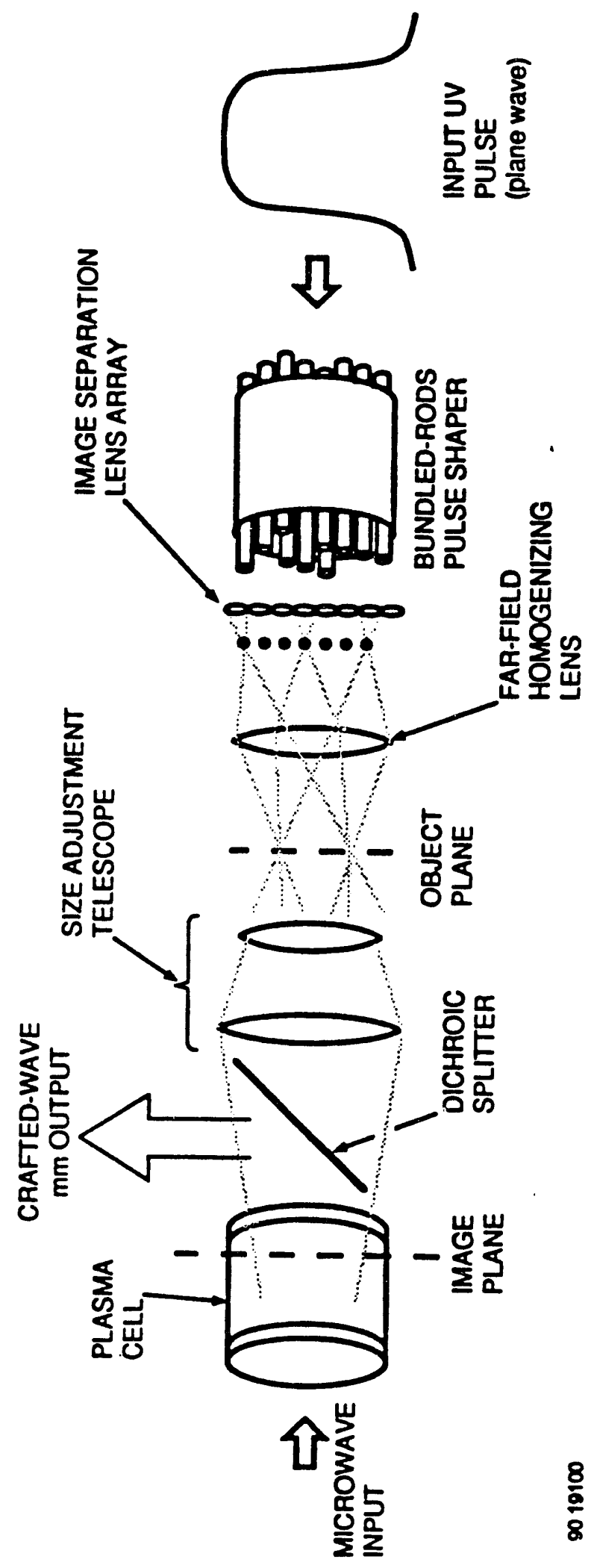

:

ह็

$\vec{x}$

$\infty$

姁

空

ह

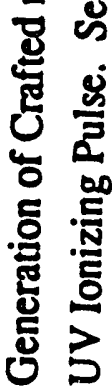

نั่ 
communications, but now extremely rapid and intensive development is underway across a broad range of smart munitions and munitions delivery systems. This inclucies the various aircraft, ship, and battlefield missiles which have onboard tracking capability.

Most of the high growth area in millimeter and microwaves can be traced to the development of IMPATT diode sources. The IMPATT is employed in the majority of applications where size, weight, cost, and reliability are factors and where low power is acceptable. Areas without these criteria, such as plasma diagnostics with millimeter waves, has seen less dramatic growth in recent years. Nearly all of the non-research applications are found at $95 \mathrm{GHz}$ or below, and not surprisingly, the maturity of component technology falls rapidly above that point.

The $95 \mathrm{GHz}$ frequency corresponds to one of the atmospheric windows that are seen in Figure 3-3. Here the dominant frequencies of interest are seen to be centered around 35, 94, 140, and $220 \mathrm{GHz}$. These frequencies of course correspond to low absorption. The molecular oxygen absorption peak at $60 \mathrm{Ghz}$ is also of special interest, precisely because of the absorption; it allows a secure communications with limited opportunity for interception. The precise attenuation desired can be controlled by variation of frequency within the wing of the absorption feature.

\title{
Table 3-1 \\ DOMINANT USES OF THE MIIIMETER AND MICROWAVE SPECTRUM
}

\author{
Instrumentation/Diagnostics \\ Electronics \\ Plasma \\ Gas \\ Basic Physics \\ Spectroscopy \\ Device Characterization \\ Radiometry \\ Meteorological Measurements \\ Astronomical \\ Radar \\ Meteorological \\ Atmospheric \\ Astronomical \\ Aircraft \\ Ship \\ Automotive
}




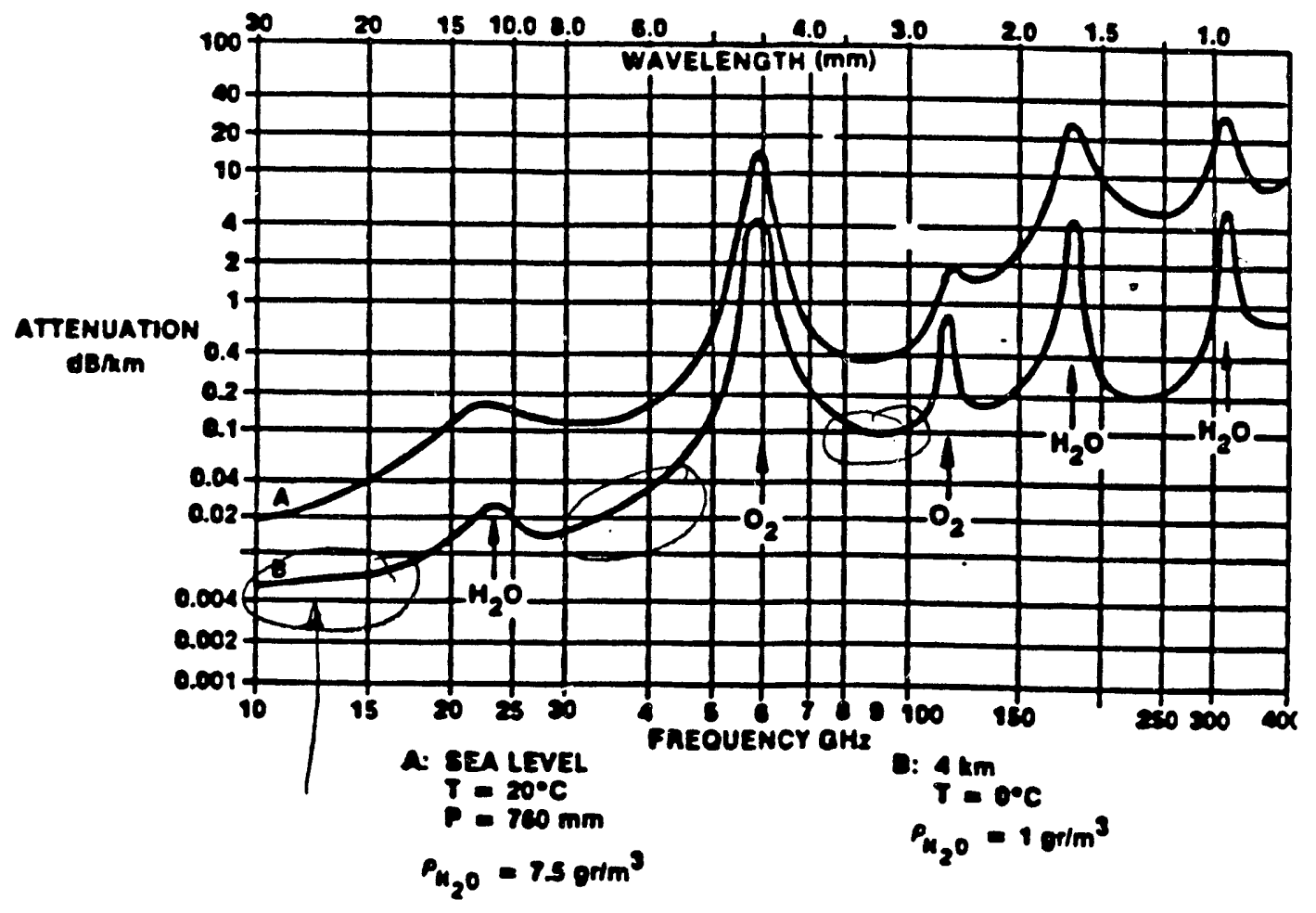

Figure 3-3. Atmospheric Absorption of mm Waves, taken from Reference 13.

11

1

?

1

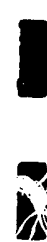




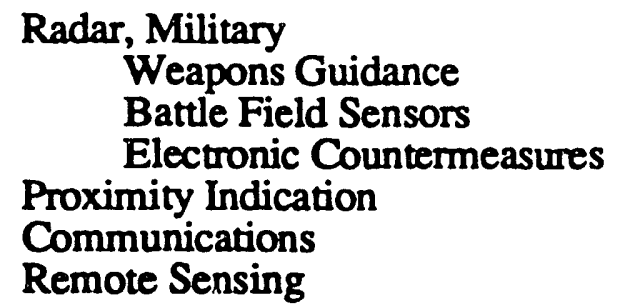

Many of the applications of Table 3-1 have been developed to use narrow band sources, some with temporal pulsed format and some continuous wave. They are not, in their present embodiment, particularly well matched to the capabilities of newer broadband sources, including the plasma wave upshifter. A second application list is given in Table 3-2 to specifically identify the potential for wide-band and tunable pulsed sources.

Table 3-2

APPLICATIONS REGUIRING BANDWIDTH, TUNABIITY, AND MODERATE POWER

\section{High Power Component Development}

General lack of high performance components for $\mathrm{mm}$ waves, existing components based on scaled microwave designs

\section{Meteorological and Atmospheric Measurements}

Multi-wavelength measurements have potential to increase information content

Radar

Multi-frequency, impulse, and crafted wave increased target discrimination capability, less susceptibility to countermeasures

Electronic countermeasures

large development effort in military spurred by measure/counter-measure competition, largely based on complex wave techniques. Also sought is means to extrapolate these techniques from microwave to $\mathrm{mm}$ 


\title{
Earth resource exploration
}

multi-frequency techniques have potential to increase information content

\section{Plasma Diagnostics}

Wide frequency range will measure larger plasma variations .

Gas Diagnostics

Multi-frequency sensing will yield more information

\author{
Ultra Short Pulse Generation (Starting with Chirped Source) \\ Applications include basic physics and impulse radar
}

\section{High Frequency Network Analysis}

Little network analysis equipment available over $100 \mathrm{GHz}$, does not require high power 


\section{SECTION 4 \\ SOLID STATE SPECIFICS}

\subsection{INTRODUCTION}

Solid state millimeter sources (SSMS), like most solid state devices, hảve three particular advantages over "tube" type millimeter sources: low cost, high reliability, and simplicity of operation. Due to their low power capability, SSMS's were confined to local oscillator type applications until after 1980 when higher power devices and suitable power combining techniques began to appear. Although SSMS's have low power capability, there are many applications which do not need high power but require small, lightweight devices. For higher power applications, solid state device will not be able to compete until phased array techniques are extended to millimeter waves.

Figure 4-1 shows a comparison between the three types of millimeter sources: solid state, vacuum electronic (tube), and quantum effect devices (lasers). It can be seen by this figure that solid state devices cannot compete with other sources in the millimeter band ( $30-300 \mathrm{GHz}$ ) for high power (> 100 Watts) applications.

There are three broad categories of solid state millimeter sources: two terminal devices, three terminal devices, and harmonic generators or frequency multipliers. Two terminal devices are basically negative differential resistivity devices (IMPATT, Gunn, etc.). Three terminal devices have basic transistor structures (FET, Bipolar, etc.). Harmonic generators or frequency multipliers, in the millimeter band, most typically, use a nonlinear capacitor (varactor) or nonlinear inductor (ferrite). The highest power devices, to date, are the two terminal devices such as the IMPATT or Gunn device. Figure 4-2 shows a comparison between several solid state devices. Frequency multipliers are not shown in Figure 42 because the demonstrated output powers have been on the order of a few to tens of milliwatts.

Figure 4-3 shows a qualitative comparison of several solid state millimeter sources. While IMPATT devices have higher power capabilities at high frequencies, Gunn devices have a much better noise figure at medium powers and FETs are probably best suited for low frequency, low noise applications. 


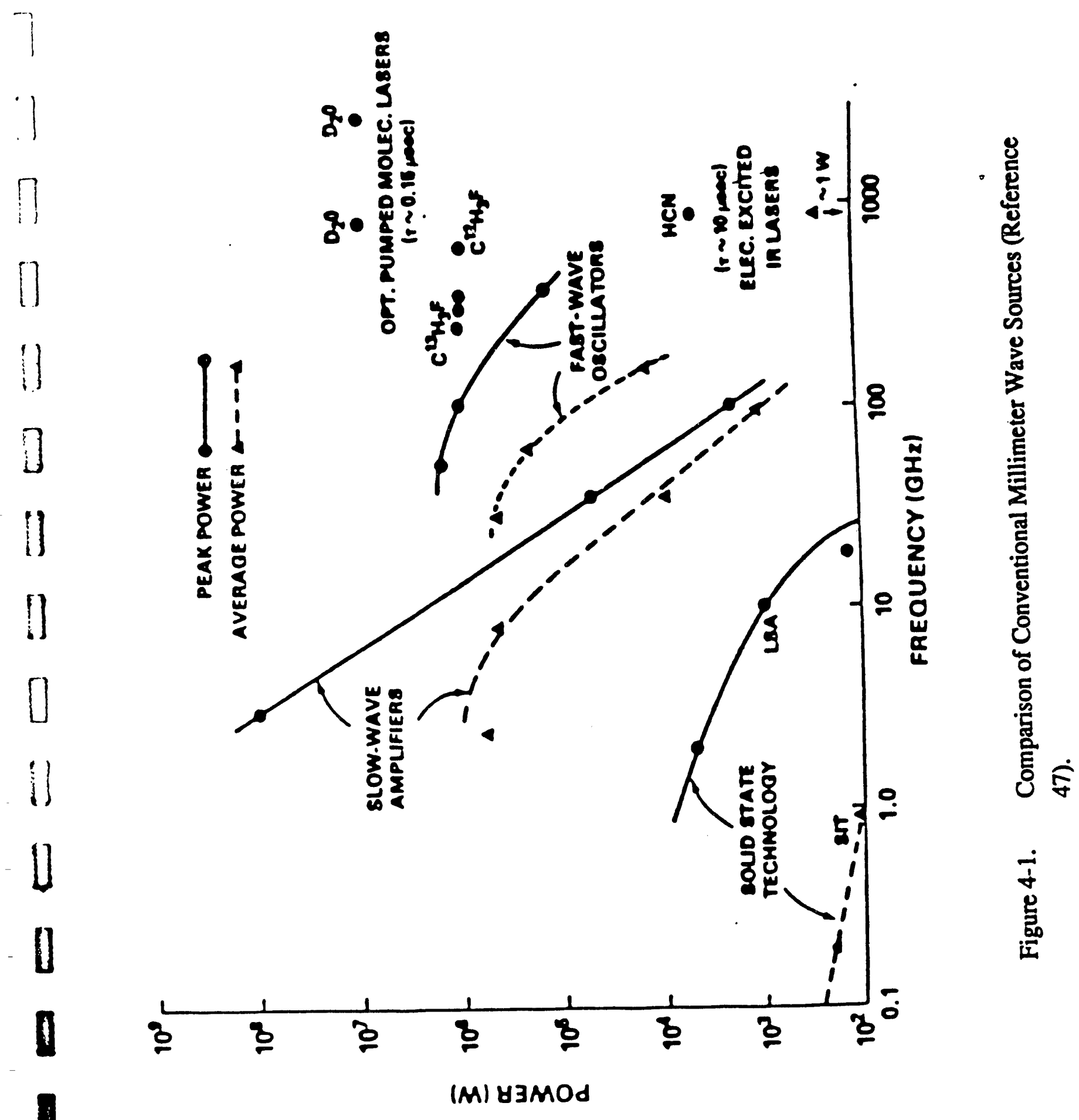




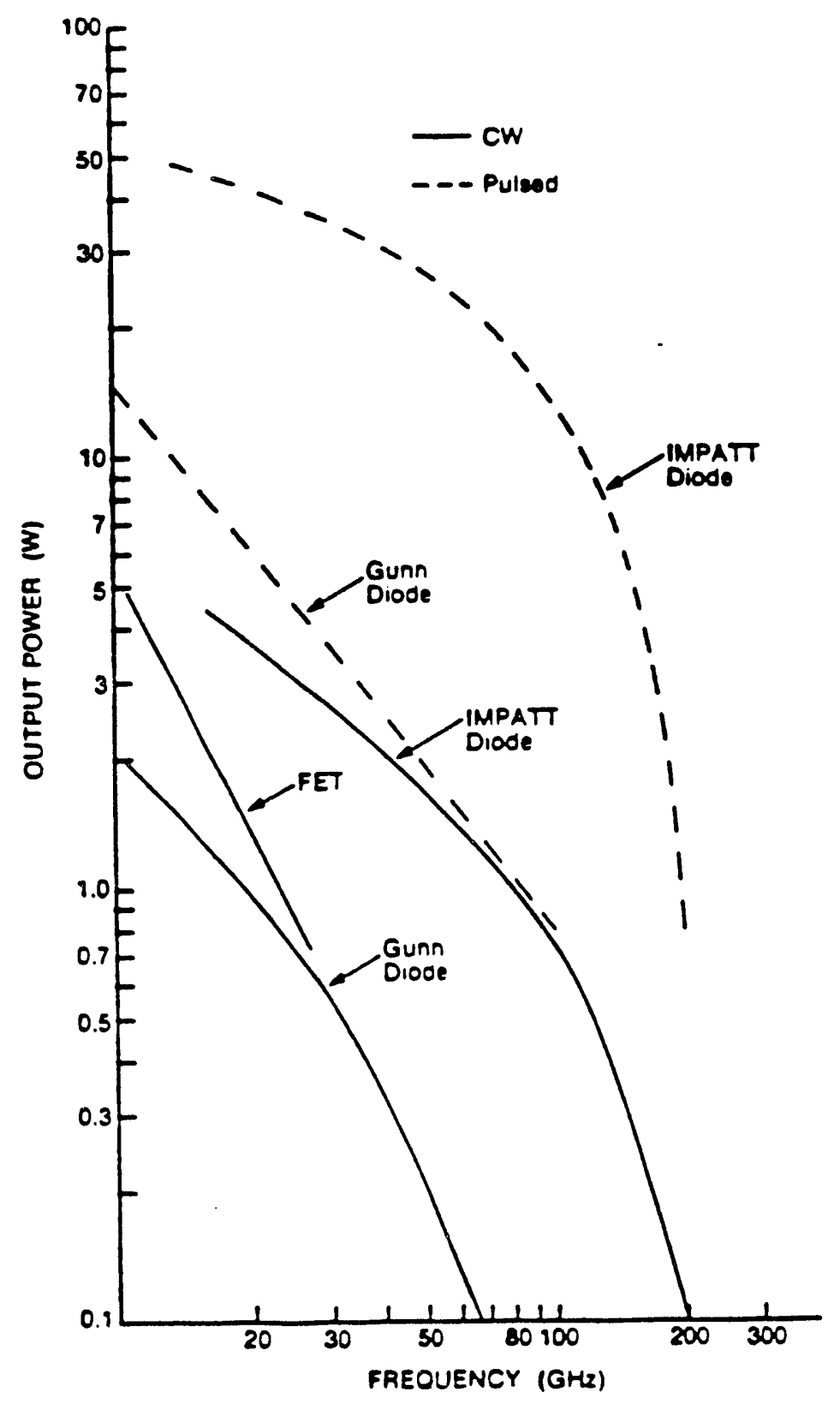

Figure 4-2. Comparison of Solid State Sources (Reference 31). 
Characteristic Amplifier Type

Operation frequency

FET < BARITT < Gunn < IMPATT

Bias voltage FET < Gunn < BARITT < IMPATT

Efficiency BARITT < Gunn < IMPATT < FET

Noise figure FET < BARITT < Gunn < IMPATT

Power BARITT < FET < Gunn < IMPATT

Gain $\times$ bandwidth BARITT < IMPATT < Gunn < FET Stability BARITT < IMPATT < Gunn < FET

Figure 4-3. Qualitative Comparison of Solid State Source Characteristics (Reference 31). 
Figures 4-4 and 4-5 show two methods of using semiconductor devices for millimeter sources. Figure 4-4 shows application of a two terminal device in an oscillator configuration. When a voltage is applied to the device, it begins to oscillate at the frequency defined by the resonant cavity and the device parameters. Figure 4-5 shows how such a device could used for amplification of a signal. It can be noted here that solid state devices are low voltage devices (few volts) and the two terminal devices require a bias voltage to operate.

This paper will review several notable device structures, their respective capabilities, and most typical applications for the devices. A short review of power combining techniques will be given. And, finally, some information about manufacturers of these devices will be presented.

\subsection{IMPATT DIODES}

The IMPATT diode is perhaps the most common of the two terminal devices. As noted in the introduction, IMPATT's can produce higher powers at the high end of the millimeter band than any other solid state device. The word IMPATT is an acronym for "impact ionization avalanche transit time". MMPATT diodes use both avalanche and transit time effects to produce a negative differential resistance.

Negative differential resistance arising from transit time in semiconductors was first considered by Shockley in 1954 [ref 24]. The first observation of the IMPATT oscillation was reported in 1965 [ref 25]. Since that time, IMPATT devices have developed into the most powerful of the solid state millimeter devices. Although they are capable of high power operation, IMPATT's produce a relatively large amount of noise and require great care in circuit design to avoid detuning or burnout [ref 26].

A number of different IMPATT effect devices have been developed but all have basically the same operational physics. Typically, the device is biased slightly into avalanche breakdown by an external voltage. Avalanche electrons, which are generated near the $\mathrm{p}^{+}$region, drift across the $\mathrm{n}$ region with a transit time determined by the device parameters. This transit time causes the current to lag the voltage, and oscillation is possible if the delay exceeds one quarter cycle [ref 27]. This transit time effect associated with the avalanche electrons give rise to the negative differential resistivity. In oscillators (amplifiers) the oscillations begin with thermal noise (signal) and is sustained by the negative differential resistivity effect combined with the resonant circuit. 


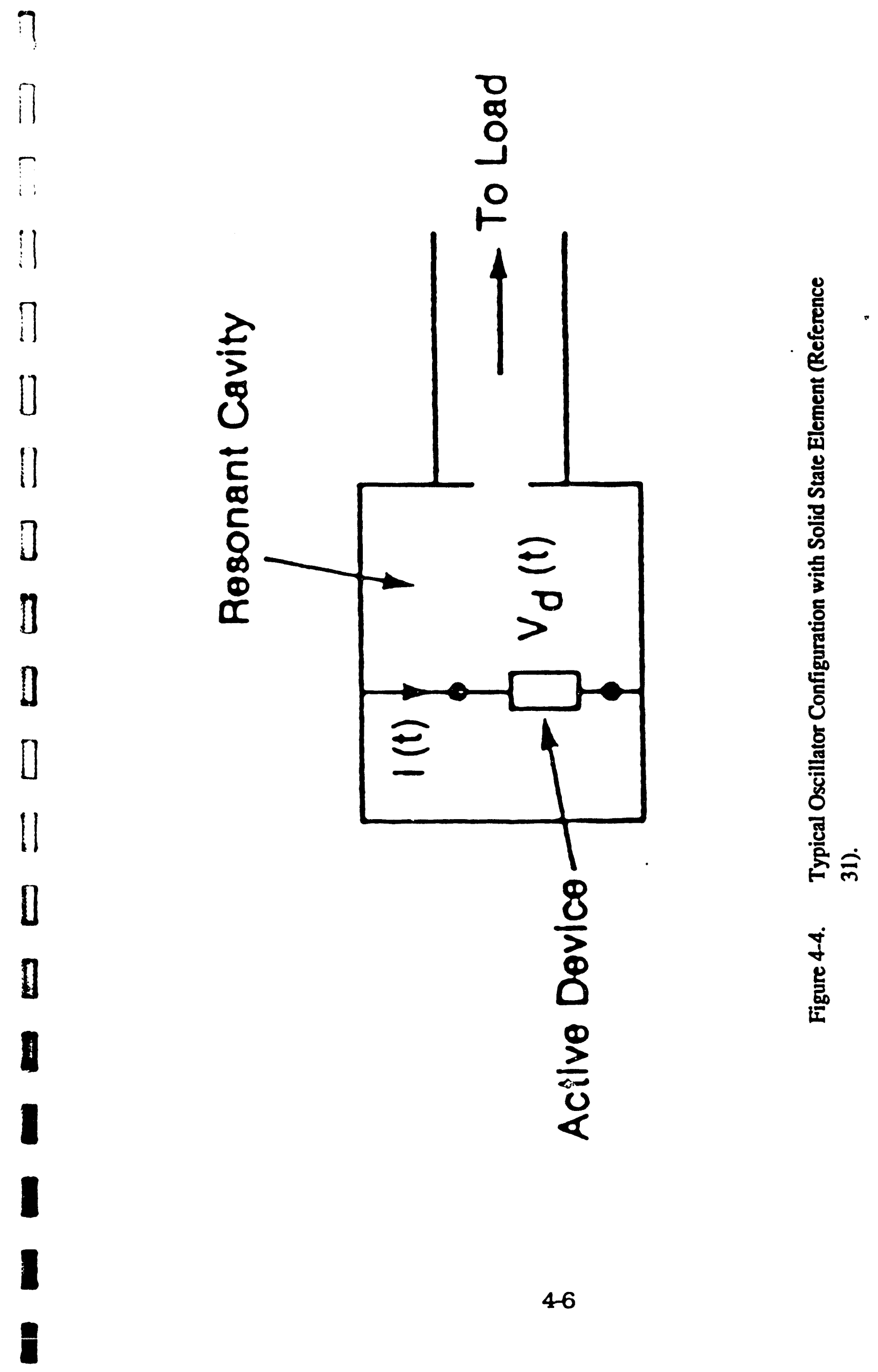



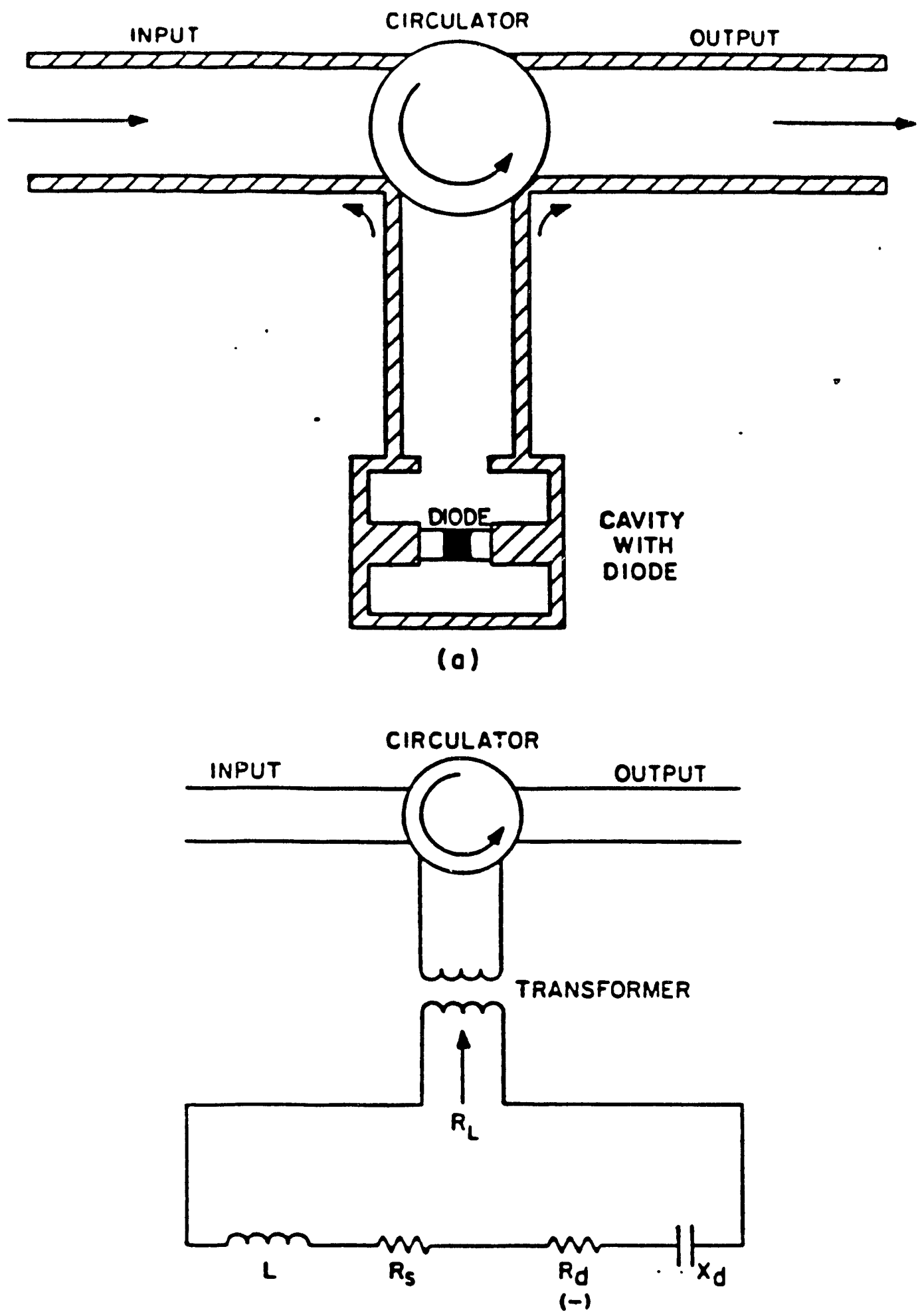

(b)

Figure 4-5. Typical Amplifier Configuration Utilizing Solid State Element (Reference 28). 
There are many manifestations of the IMPATT effect in semiconductor devices. The basic structures include: Read diode, one-sided abrupt p-n junction, two-sided (double drift) diode, hi-lo and lo-hi-lo diodes (modified Read diode), and p-i-n diode (Misawa diode). Figure 4-6 shows two types of IMPATT structures: the single-drift or one-sided and the double-drift or two-sided diodes. In the one-sided structure, electrons carry the current. This allows the device to operate at very high frequencies due to the high mobility of electrons. The two-sided structure uses both holes and electrons to carry current and is, therefore, more efficient at lower frequencies because of the greater current density capability. Details of the physics of these devices can be found in references $28-30$.

IMPATT devices are capable of operating over a frequency range which theoretically can extend up to $1000 \mathrm{GHz}$ although realistic (useful power) operation can only be expected up to about $300 \mathrm{GHz}$. For $\mathrm{CW}$ devices, thermal issues require diamond heat sinks at frequencies above $100 \mathrm{GHz}$ (Power limit scales like $1 / \mathrm{f}$ ). For both pulsed and $\mathrm{CW}$ devices, high frequency operation is limited by the critical electric field and the saturation velocity (Power limit scales like $1 / \mathrm{f}^{2}$ ). Figure 4-7 and Figure 4-8 shows the power capability of IMPATT devices as a function of frequency for pulsed and CW devices respectively. Note the $1 / \mathrm{f} \mathrm{thermal} \mathrm{limit} \mathrm{at} \mathrm{low} \mathrm{frequencies} \mathrm{and}$ the $1 / \mathrm{f}^{2}$ electronic limit at higher frequencies.

The vast majority of devices fabricated to date have been made of silicon. Silicon devices are most poplar because they are based on an extensive data base of knowledge for the technology. GaAs devices have been built but appear to be limited to lower frequencies.

As can be seen in Figures 4-7 and 4-8, the highest power available from a single pulsed device (in the millimeter band) is about 30 watts and the highest available power from a CW device is about 2 watts. Power combiners have been used in both pulsed and CW applications. The highest pulsed power available from an IMPATT combiner is 63 watts (at $94 \mathrm{GHz}$ ) and 10 watts of power has been obtained by a CW combiner.

The efficiency of practical devices is controlled by a number of issues: space charge effect, reverse saturation current, series resistance, skin effect, saturation of ionization rate, tunneling effect, intrinsic avalanche response time, and minority carrier storage effects [ref 25]. While it is beyond the scope of this paper to address all of these issues, Figure 4-9 indicates the sum effect of these factors. Figure 4-9 shows 


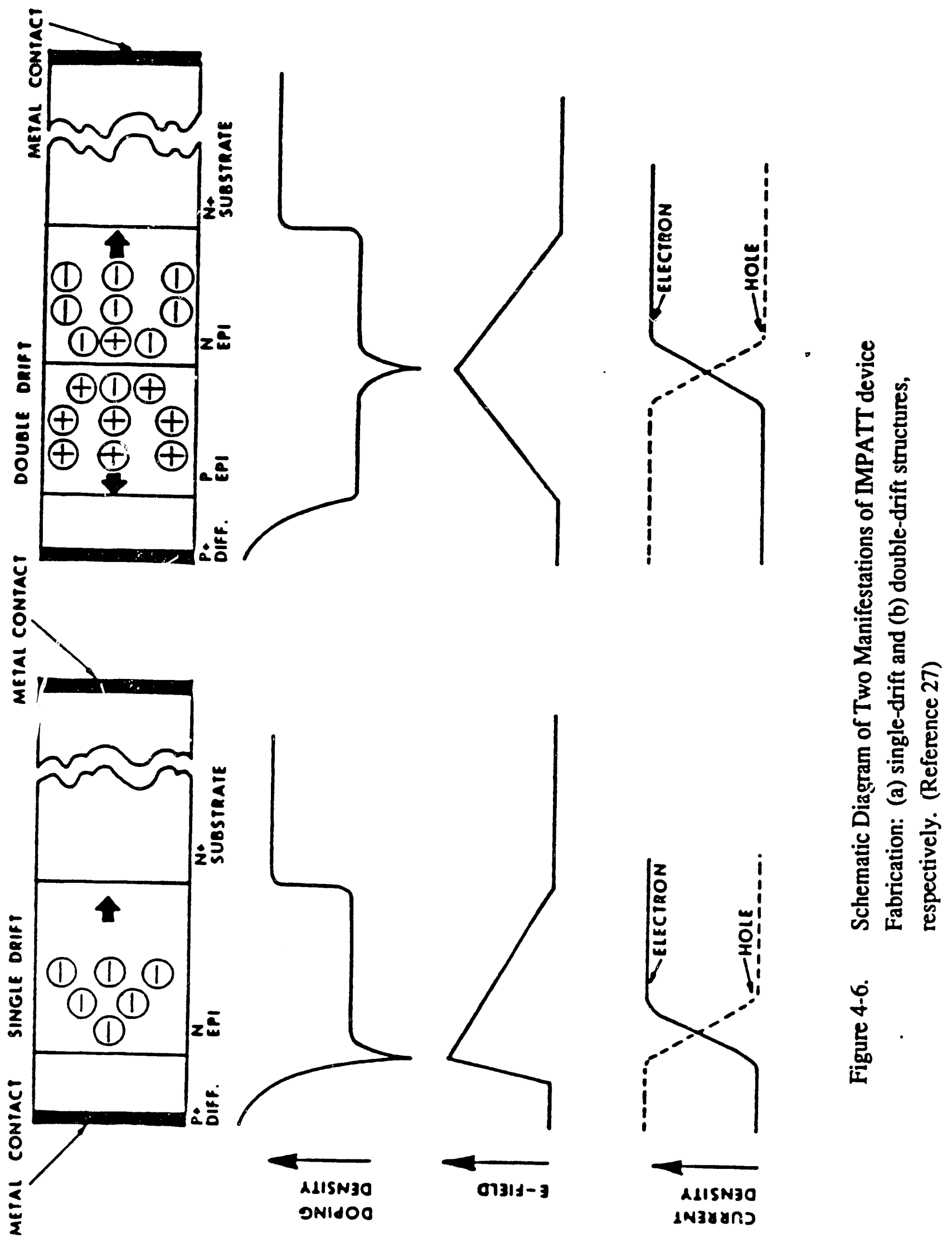


1

1

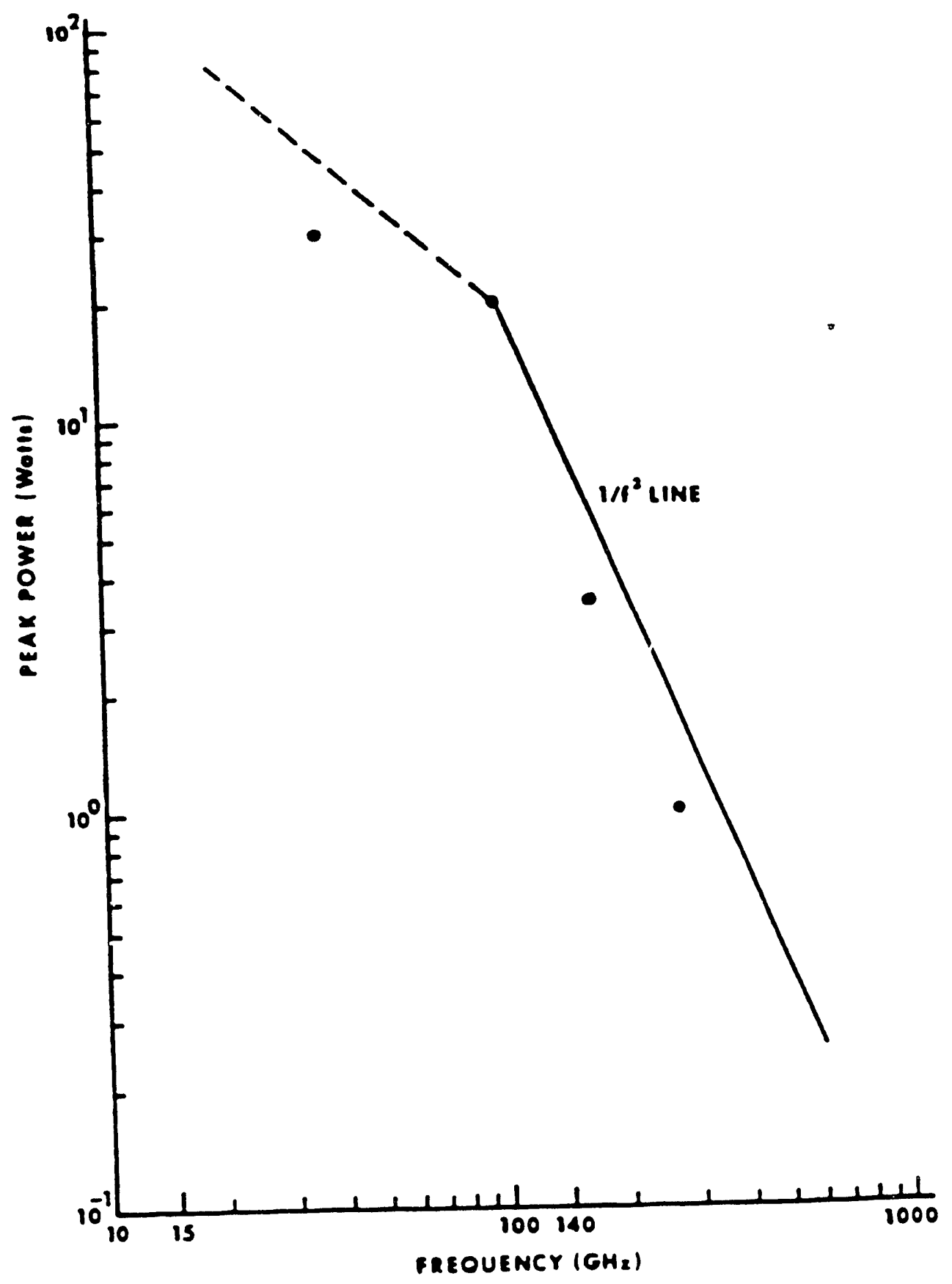

[]

i)

if

11

19

$\left.\begin{array}{l}7 \\ \vdots \\ 3\end{array}\right]$

1

1

I

1

1

Figure 4-7. Current Technology in Pulsed IMPATT Power Capability Based on Data from Hughes, Plessey and Raytheon (Reference 27). 


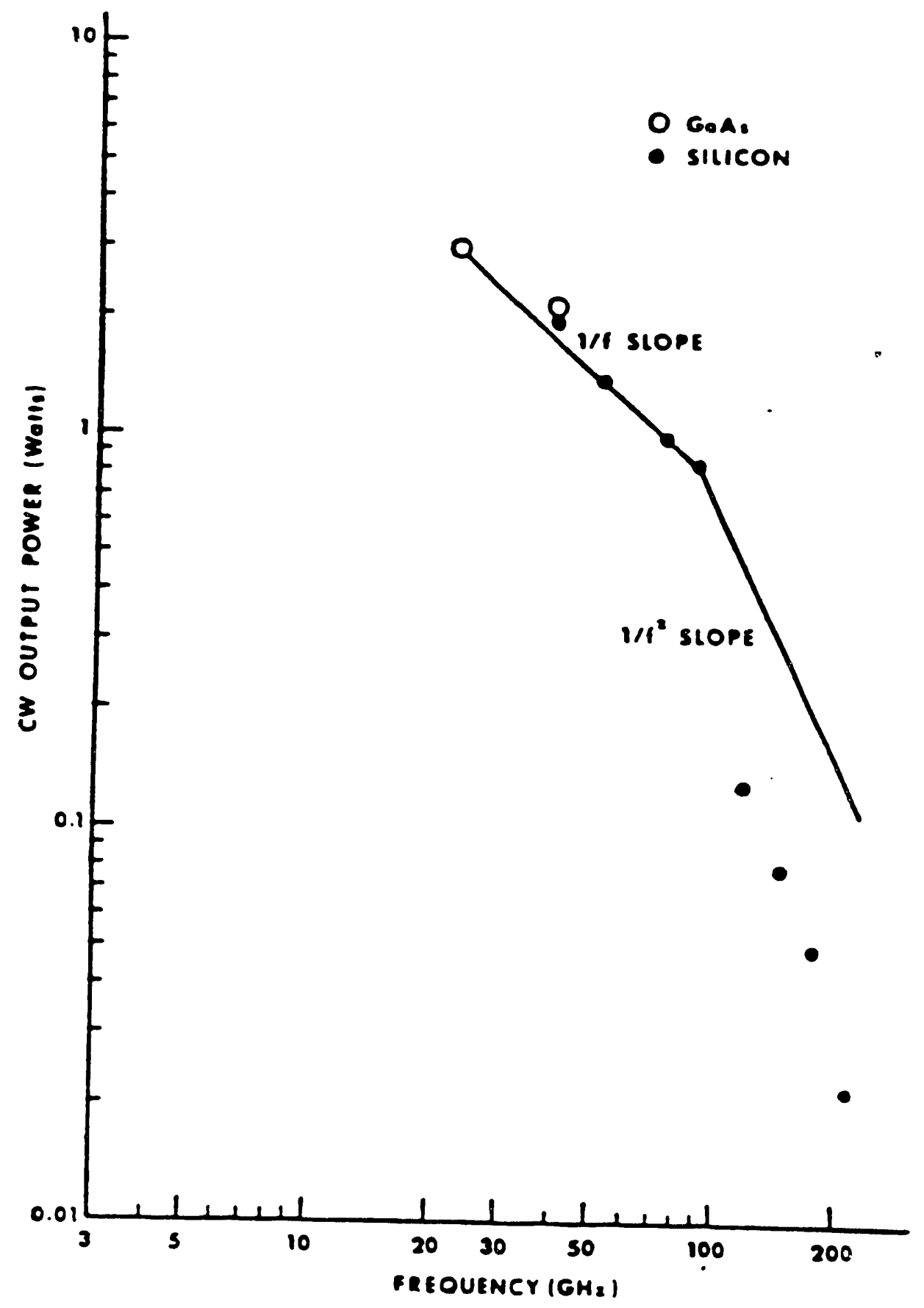

Figure 4-8. CW IMPATT Power Capability Based on Data from Hughes, Plessey, and Raytheon (Reference 27). 


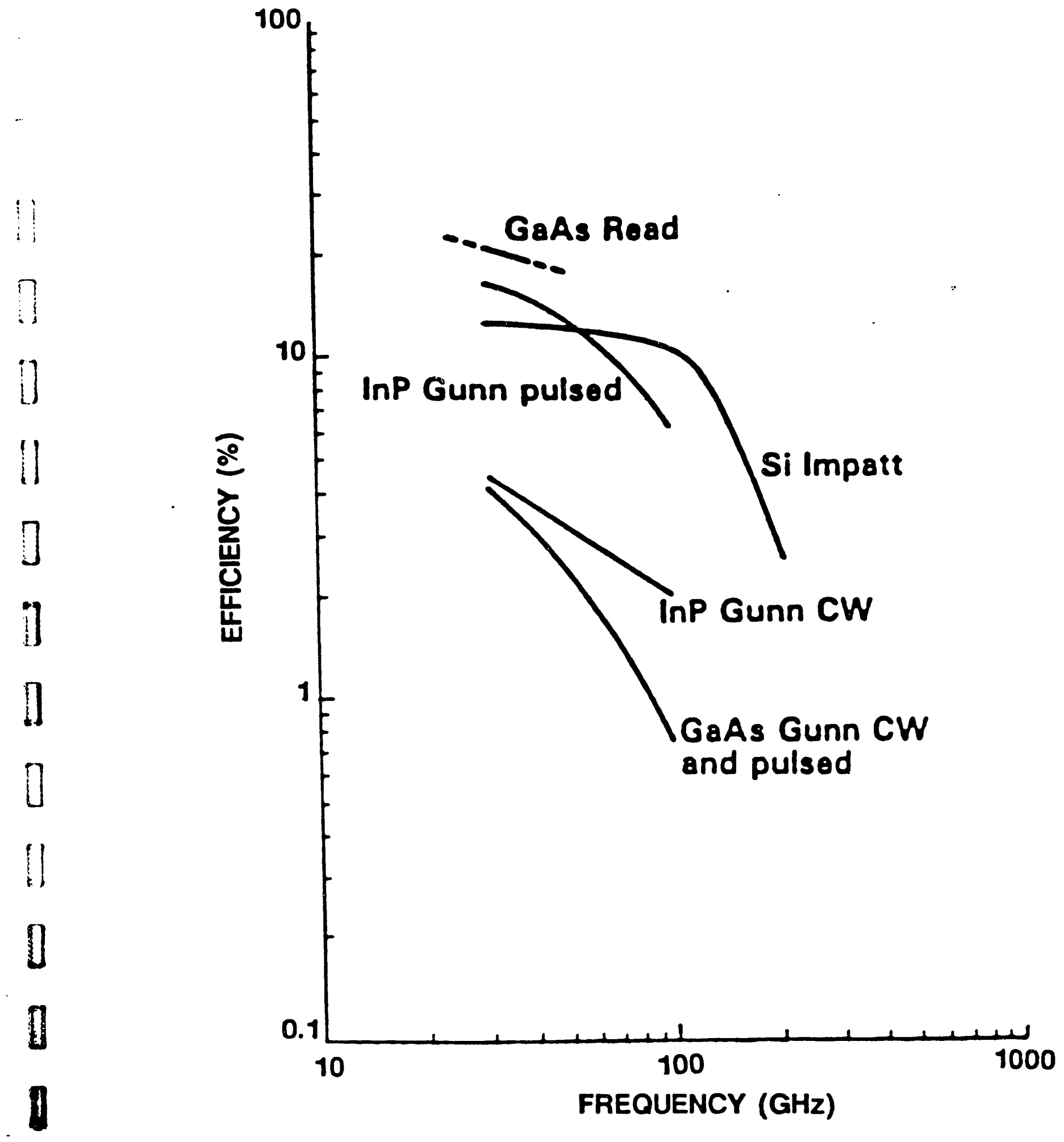

Figure 4-9. Efficiency of Selected Solid State Devices (Reference 48). 
the efficiency of both GaAs and silicon IMPATT devices (as well as Gunn devices) as a function of frequency. Again, GaAs devices have not been fabricated for frequencies which extend very far into the millimeter band. Silicon devices have an efficiency of around 10 percent for frequencies between 30 and $100 \mathrm{GHz}$. After 100 $\mathrm{GHz}$, the efficiency begins to roll off very quickly.

Because the physical process in an IMPATT diode is an avalanche effect, MPATT diodes have a relatively high amount of noise. This noise is due to the statistical nature of the avalanche process. This limits the signal input level to the diode in an amplifier configuration. GaAs devices have been found to have significantly less noise than silicon devices [ref 28].

Frequency stability for IMPATT oscillators is typically on the order of $-5 \times 10^{-5}$ per degree Celsius. Power output variation is on the order of $-0.005 \mathrm{~dB}$ per degree Celsius. This amount of frequency variation is not acceptable for applications such as coherent and Doppler radars, molecular astronomy, communications, spectroscopy, and instrumentation without some method of stability improvement [ref 31]. Methods are available which allow the frequency stability to be improved. These methods include: cavity stabilization, injection locking, and phase locking. Cavity stabilization is generally very difficult and expensive at millimeter wavelengths. Several cavity techniques are practiced and include: cavity temperature control, high $Q$ cavities, frequency discrimination (filtering), and automatic frequency control. Injection locking is a popular technique. Using this technique, frequency stabilities on the order of the injector stability can be achieved although the oscillator must have a low $Q$. Phase locking combines the advantages of both cavity stabilization and injection locking without the low $Q$ requirement. Phase locking can be accomplished by adjusting the bias voltage or using a varactor to tune the cavity.

Chirp occurs in IMPATT's due to junction temperature changes during operation. This can be compensated by ramping the applied device current in the right proportion to balance the heating effects.

The frequency of IMPATT oscillators can be varied over a relatively wide range. Varactor diodes can be used to vary the operating frequency up to about $50 \mathrm{GHz}$. Varactor diodes can provide a tuning range of about $3 \mathrm{GHz}$. Above $50 \mathrm{GHz}$ tuning of the IMPATT oscillator is accomplished by adjustment of the bias voltage. Using this technique, tuning range is limited to a few percent. Mechanical 
tuning is possible, although at a much slower rate. Using mechanical tuning it is possible to tune the IMPATT over a bandwidth of 20 to $30 \%$.

Typical applications of IMPATT diodes include: phased array radar, tracking radar, active seekers, tank fire control radar, and tactical communications. In these applications, lifetimes of more than $2 \times 10^{6}$ have been predicted [ref 31 ].

Finally, Figure 4-10 shows two typical packages for IMPATT diodes. .

\subsection{GUNN DIODES}

The second most common solid state millimeter source is the Gunn oscillator. It, too, is a two port device but its physical mechanism for generating millimeter waves differs from that of the IMPATT diode. The Gunn device is based upon a bulk effect as opposed to a junction effect as in the IMPATT diodes. In 1963, Gunn discovered that coherent microwave output was generated when a dc electric field was applied across a randomly oriented, short, n-type sample of $\mathrm{GaAs}$ or InP that exceeded a critical threshold value of several thousand volts per centimeter [ref 32]. Since that time, Gunn oscillators have become well respected for their ability to produce moderate power microwaves in the millimeter band with very low noise content.

Like MMPATT diodes Gunn devices operate via the negative differential resistivity effect. Gunn devices have been placed in the category of transferred electron devices (TED) because the negative differential resistivity effect is produced by the transference of electrons from one energy state to another. This particular effect is called the Gunn effect or the Ridley-Watkins-Hilsum effect. Basically, conduction band electrons gain energy from the applied electric field and transfer from a low energy, high mobility state to a high energy, low mobility state (see Figure 4- 11). This reduction in carrier mobility with increased electric field gives rise to the negative differential resistance. This effect becomes significant if the applied electric field exceeds a certain threshold value. This value for $\mathrm{GaAs}$ is on the order of $3-5 \mathrm{KV} / \mathrm{cm}$ and for InP it is about $10 \mathrm{KV} / \mathrm{cm}$. Neither silicon nor germanium exhibit this behavior.

Although Gunn devices are capable of producing moderate millimeter band powers, most designers tend to use these devices for local oscillators in receivers because of their extremely low noise content. Figure 4-12 shows a comparison of noise measures for several semiconductor devices. Although the noise figures for 


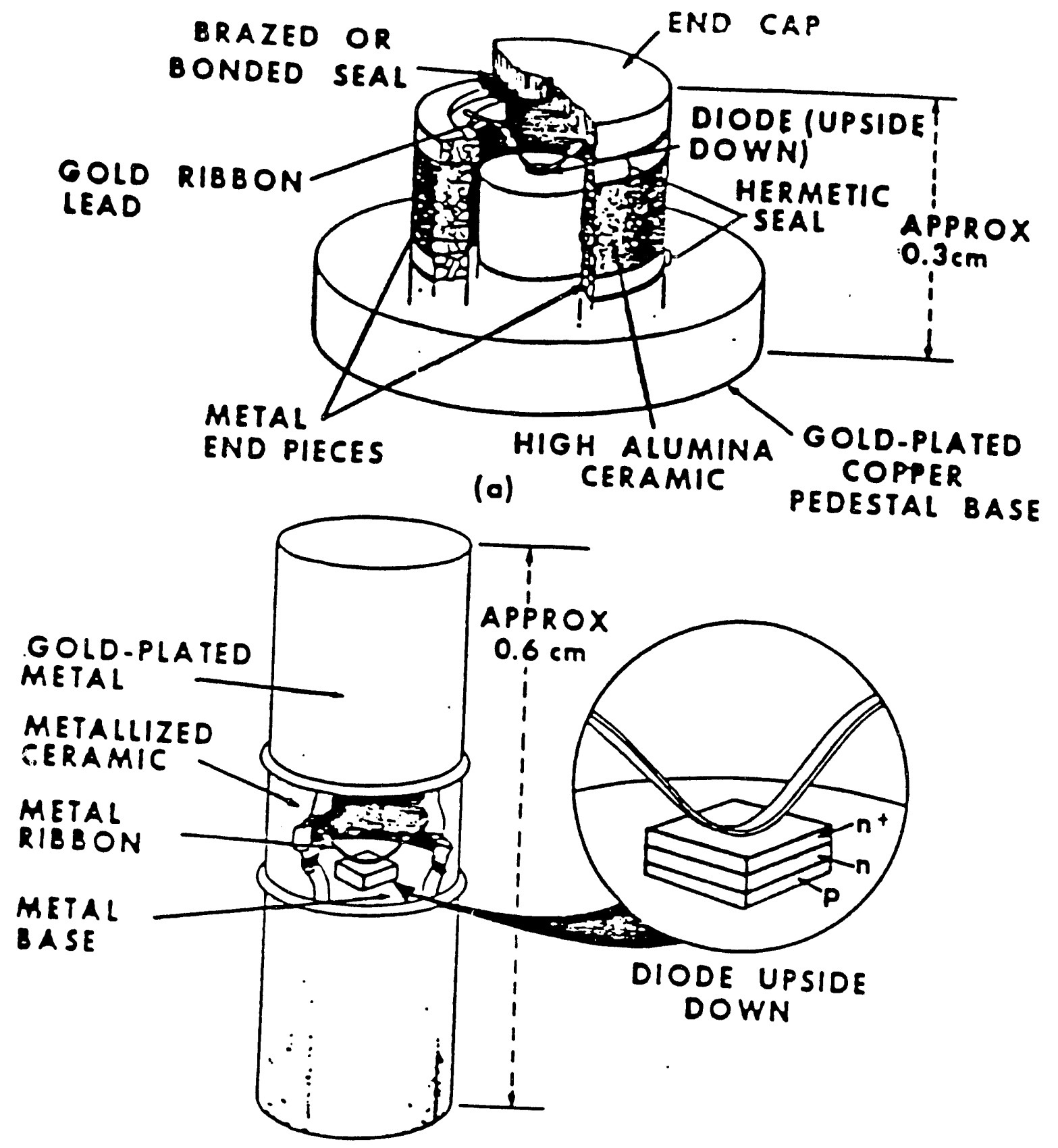

(b)

Figure 4-10. Typical Packages for IMPATT Diodes (Reference 28). 
7

.1

$\left.\begin{array}{l}7 \\ \vdots\end{array}\right]$

I

1

U

1

目

$-$

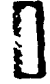

$-11$

D

0

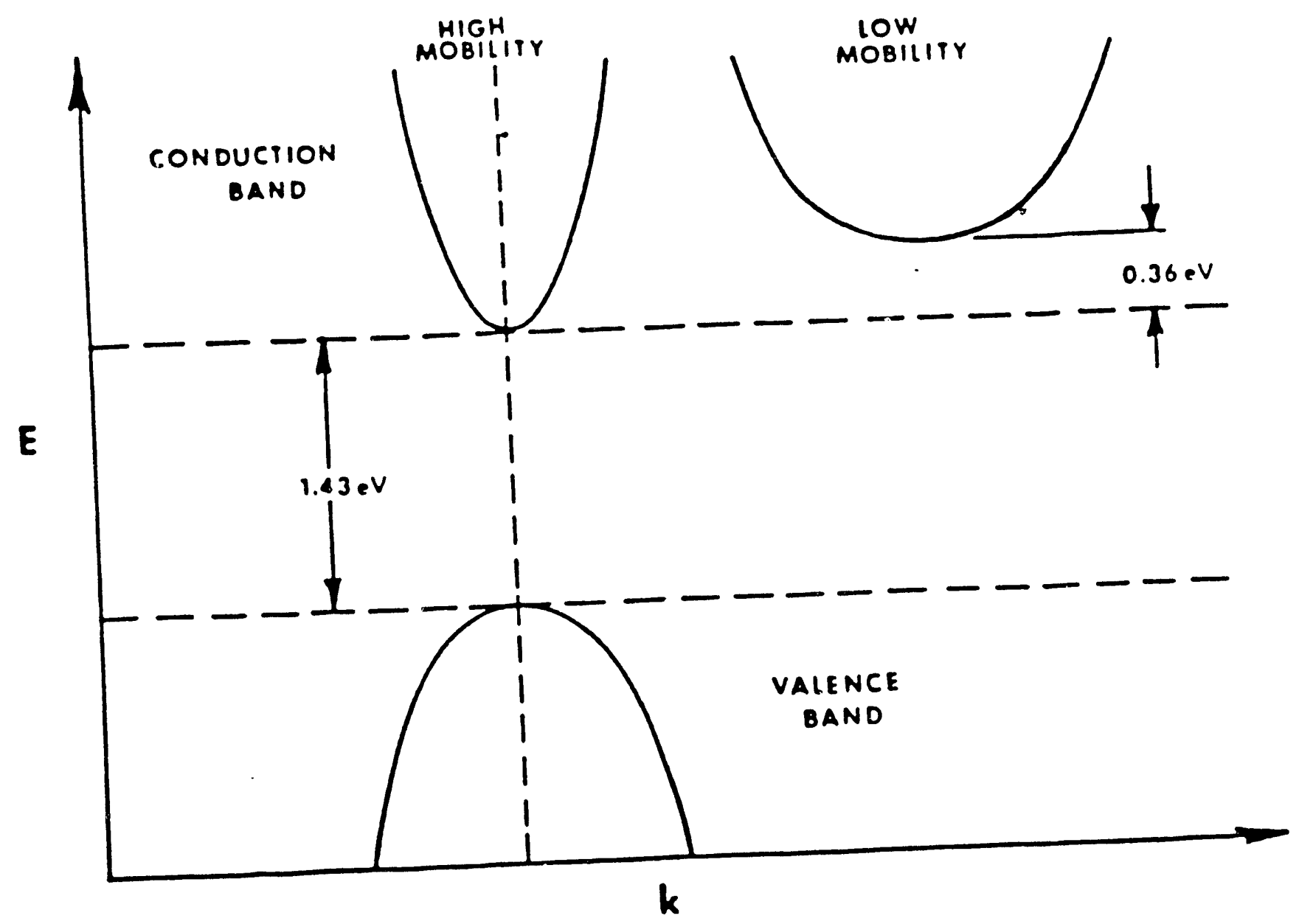

Figure 4-11. Simplified Schematic Diagram of the Energy Levels in a Gunn Oscillator, Negative Resistance Results When Carriers are Excited Into the Higher Energy, Low Mobility Level (Reference 31). 


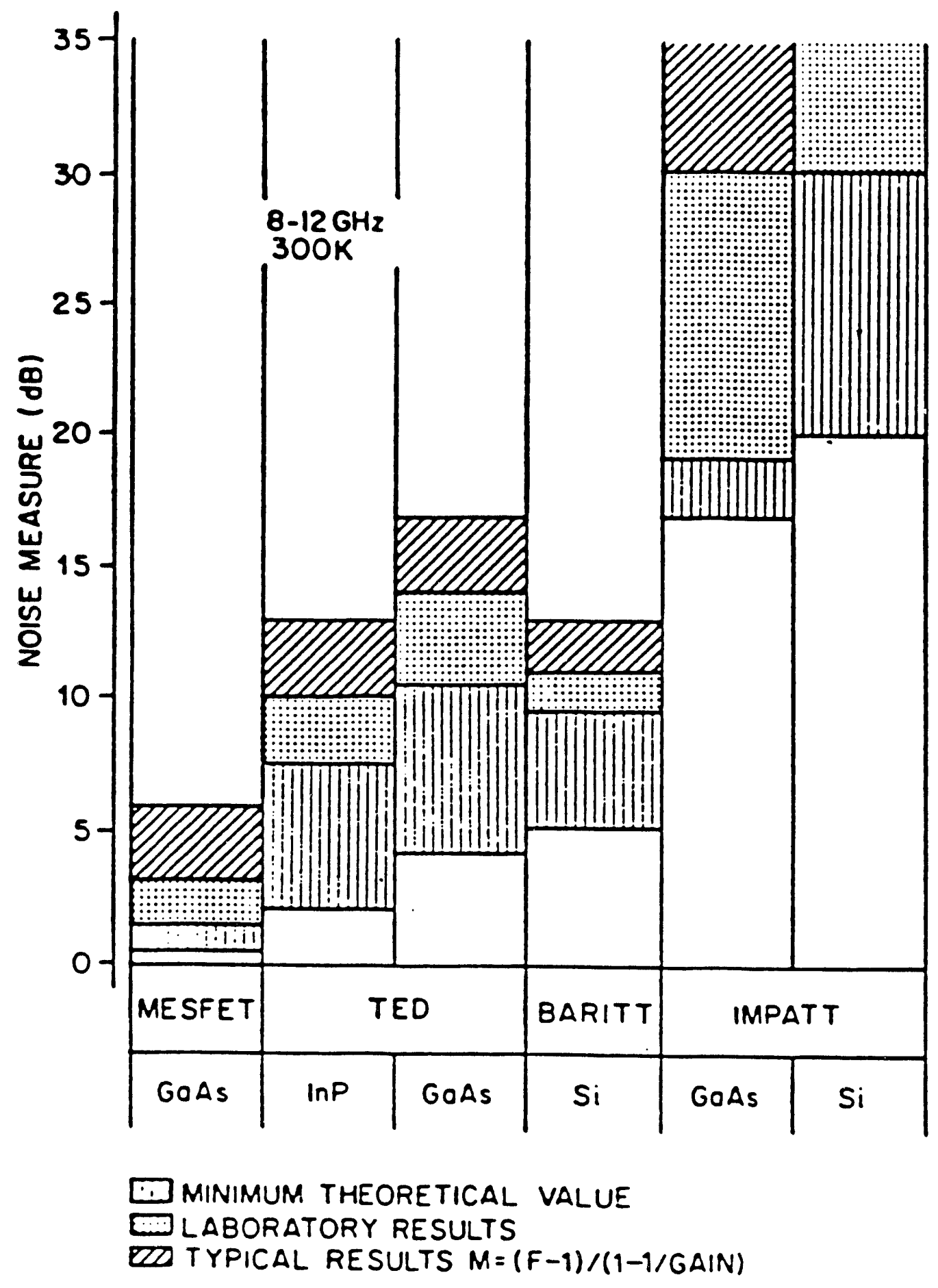

Figure 4-12. Comparison of Small-Signal Noise Measures for Various Semiconductor Microwave Devices in the Frequency Range 8 to 12 $\mathrm{GHz}$ (Reference 28). 
Figure 4-12 are for frequencies up to $12 \mathrm{GHz}$, it does give a relative indication of the noise performance of Gunn (TED) devices compared to IMPATT diodes.

Gunn devices are capable of operating over a wide range of frequencies. GaAs devices are capable of operating up to $100 \mathrm{GHz}$. Operation above this frequency is limited by the carrier mobility. InP devices appear to have an upper frequency limit of $200 \mathrm{GHz}$ because of the higher mobility of carriers in InP. At lower power levels, Gunn devices can be used with frequency multipliers to go as high as $230 \mathrm{GHz}$. This ability to operate with frequency multipliers is due to the very low noise of Gunn devices. Figure 4-13 shows the power capability as a function of frequency for pulsed and $\mathrm{CW} \mathrm{GaAs}$ devices and InP devices. Pulsed GaAs devices have operated up to 20 Watts at $30 \mathrm{GHz}$ ( 400 milliwatts at $90 \mathrm{GHz}$ ) and up to 400 milliwatts at $30 \mathrm{GHz}(70$ milliwatts at $95 \mathrm{GHz}$ ) in a $\mathrm{CW}$ mode. InP devices have operated in a CW mode at power levels up to 500 milliwatts at $40 \mathrm{GHz}$ ( 100 milliwatts at $100 \mathrm{GHz}$ ). Pulsed InP devices are capable of producing 6 watts of power at $30 \mathrm{~Hz}$ and 300 milliwatts of power at $90 \mathrm{GHz}$.

Figure 4-9 shows the efficiency of Gunn devices. The energy relaxation time due to collisions and the acceleration-deceleration time required for an electron to gain or lose the energy separating the energy levels are lower by a factor of two for InP than for GaAs. This should make the InP devices about twice as fast or the frequency limit about twice as high as GaAs. Also, the thermal conductivity of $\mathrm{InP}$ is $25 \%$ greater than GaAs. This means that the device can operate at lower temperatures. In general, InP oscillators can produce a factor of two higher powers than $\mathrm{GaAs}$ and the efficiency of InP devices is a factor of three higher. Nevertheless, Gunn devices are not as efficient as IMPATT devices at the same frequency except at the lowest frequencies in the millimeter band.

Operation of these devices are the same as IMPATT devices as far as physical (circuit) configuration is concerned (see Figures 4-4 and 4-5) Furthermore, the comments relative to IMPATT tuning ability applies to these devices, also. Namely, for frequencies below $50 \mathrm{GHz}$ varactors can be used for a tuning range on the order of three $\mathrm{GHz}$. Above $50 \mathrm{GHz}$, bias tuning can be used to achieve a frequency range of a few percent.

Like IMPATT devices, the frequency stability of Gunn diodes is on the order of $-5 \times 10^{-5}$ per degree Celsius or $-5 \mathrm{MHz}$ per degree Celsius at $100 \mathrm{GHz}$. Stability 


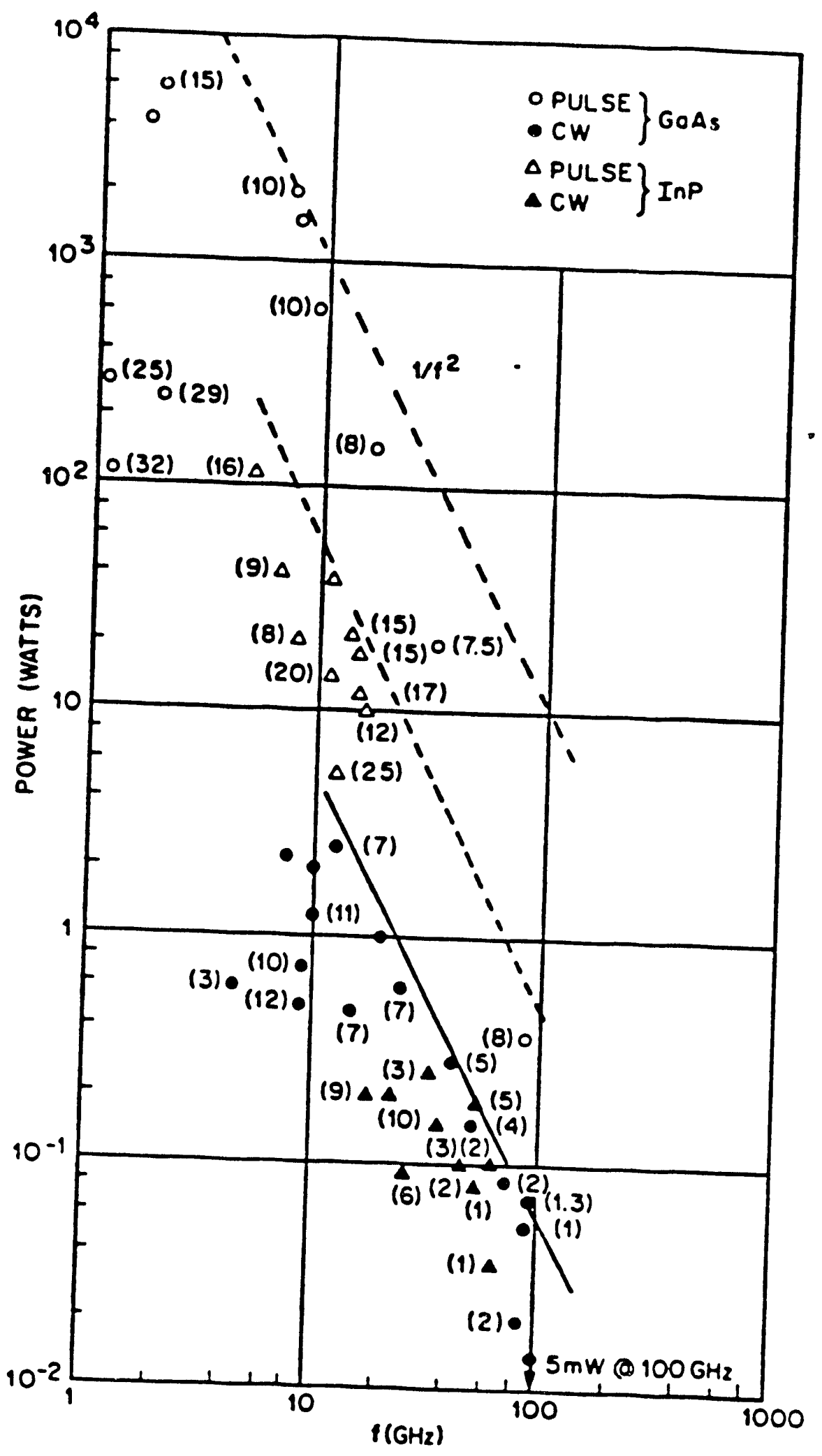

Figure 4-13. Output microwave Power vs. Frequency for Pulsed and cw-Operated GaAs and InP TEDS. The numbers in parentheses indicate the dc-to-rf conversion efficiencies in percentage (Reference 28 ). 
can, also, be improved using injection locking or phase control techniques. Power output variation for typical commercial devices is on the order of $-0.04 \mathrm{~dB}$ per degree Celsius [ref 31].

Packaging for Gunn devices is similar to that of IMPATT devices (see Figure 4-10). Fabrication of Gunn diodes is less complicated than IMPATT devices (because they are bulk devices as opposed to junction devices) and are, therefore, less expensive, although, the Gunn bulk material must be very pure and free from defects.

\subsection{TUNNEL DIODES}

Although IMPATT's and Gunn devices are the principal solid state millimeter wave sources, several other devices have been used to some degree for millimeter wave applications. The tunnel diode is another two terminal negative differential resistivity device used to generate millimeter waves. Tunnel diodes were discovered in 1958 by Esaki [ref 33] and were actually the first negative resistance active semiconductor device used at microwave frequencies [ref 34]. While limited in power to a few milliwatts, these devices found use as low noise amplifiers. Since the development of Gunn and DMPATT sources tunnel diodes have received little attention.

A tunnel diode is a negative resistance device which consists of a simple heavily doped p-n junction. Figure 4-14 shows the static voltage and current relationships for a tunnel diode. As a forward bias voltage is applied to the diode, electrons begin to tunnel from the $n$ side of the junction to unoccupied states on the $p$ side. As the voltage is increased further, there are fewer unoccupied states on the $p$ side so current decreases. As the voltage is increased further still, the current due to tunneling goes to zero and the current which flows is due to normal thermal current flow. The decline in current with increased voltage gives rise to the negative differential resistance effect.

Oscillations in tunnel diode circuits have been reported up to $103 \mathrm{GHz}$ at power levels of microwatts [ref 35]. Although tunnel diode technology is not considered viable for generation of millimeter waves (because of low power levels), the diodes are useful in detectors mixers, and other applications. 

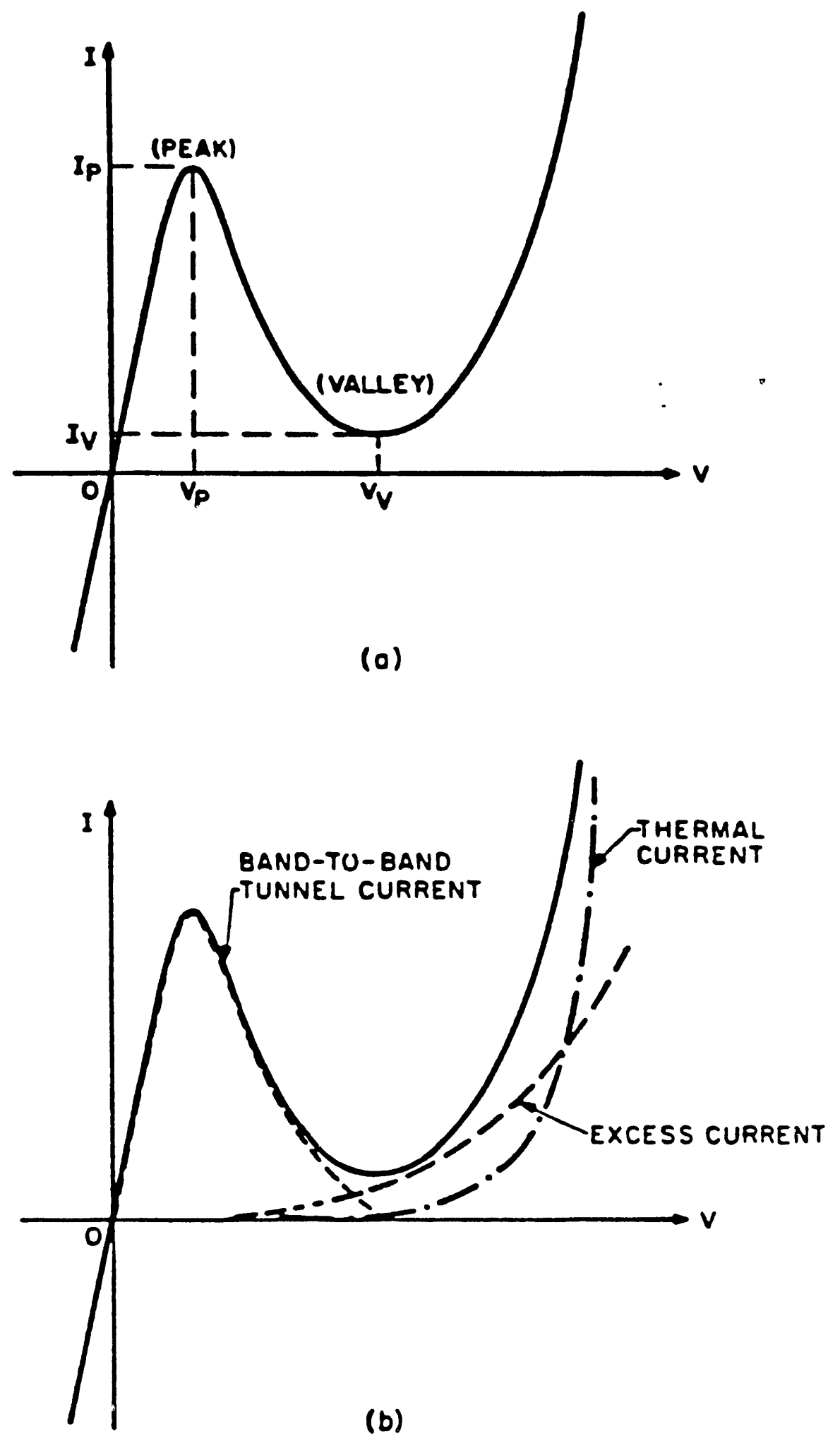

Figure 4-14. Tunnel Diode Characteristics. (a) Static current-voltage characteristics of a typical tunnel diode. $\mathrm{I}_{\mathrm{p}}$ and $\mathrm{V}_{\mathrm{p}}$ are the peak current and peak voltage, respectively. $I_{v}$ and $V_{v}$ are the valley current and valley voltage, respectively. (b) The static characteristic is broken down into three current components. (Reference 28) 


\subsection{TUNNETT DIODES:}

Because IMPATT diodes have such high noise figures, several altemative mechanisms have been proposed to reduce the noise. The TUNNETT (Tunnel Injection Transit Time) has been proposed as such a device. A TUNNETT is a device with a highly doped $p^{+}-n$ junction which relies on a tunneling process to inject carriers through the junction. From there the carriers undergo a drift or transit time effect which gives rise to negative differential resistivity. Because the carriers are injected by the tunneling process, the devices have a much lower noise figure than avalanche injected devices. Also because of the lower dispersion effect associated with tunneling injection, it is estimated that the device can be operated up to about $1000 \mathrm{GHz}$ and beyond with higher efficiencies than IMPATT diodes [ref 36].

TUNNETTS should be able to operate in the frequency range of 100 to $800 \mathrm{GHz}$. Although the TUNNETT is only a laboratory devire at this time, they have the potential of high efficiency operation at submillimeter wavelengths, possibly on the order of $5 \%$ at $500 \mathrm{GHz}$ [ref 37]. TUNNETTs have been fabricated which operate from 100 to $248 \mathrm{GHz}$ [ref 38] and $0.12 \%$ efficiency has been observed at $338 \mathrm{GHz}$ [ref 39]. TUNNETTs are difficult to fabricate because of the required abrupt junction doping profile.

\subsection{BARITT DIODES}

Barrier Injection Transit Time devices are like TUNNETTs in that they try to avoid the high avalanche noise generated in IMPATT devices. BARITT operation was first reported by Coleman and Sze in 1971 [ref 40]. These devices rely on thermal minority carrier injection (holes) and diffusion across a forward biased barrier. The BARITT device is basically a back to back pair of diodes biased into reach through condition. Because this process is not ar. avalanche process, BARITTs have a much lower noise figure than IMPATTs. BARITT diodes tend to be low in power and efficiency but have good signal to noise performance, and are useful as local oscillators in microwave receivers for use in Doppler radar applications such as intrusion alarms, industrial surveillance, and anticollision radars.

BARITT diodes have been fabricated at $29 \mathrm{GHz}$ with a power level of 4 milliwatts and an efficiency of $0.5 \%$ [ref 41 ]. 


\subsection{TRAPATT DIODES}

Trapped Plasma Avalanche Triggered Transit Time devices were first reported by Prager, et al in 1967 [ref 42]. These devices have the potential to be very high efficiency devices. In reality, they are high efficiency devices but for reactive loads only. This comment can be best understood after a brief discussion of the operation of TRAPATT's. Under ideal conditions, a negative resistance diode would produce a square wave voltage and current with both waveforms 180 degrees out of phase. The TRAPATT comes very close to this ideal situation. The device is reversed biased in to avalanche breakdown. A electron/hole plasma is formed and the voltage collapses across the device. Current flows through the device with a low voltage drop. As the plasma is swept out of the diode (by current flow), the current becomes very low and the voltage rises to a high value. Eventually the reactive energy stored in the load raises the voltage across the device until brea jown occurs and the process is repeated. Without a reactive load, the device efficiency is severely degraded.

TRAPATTs have very high noise figures but can operate at very high average powers at low frequencies. Operation of TRAPATTs appear to be limited to the lowest frequencies of the millimeter band ar best. TRAPATT amplifiers and oscillators are very complex and are not used extensively in any application.

\subsection{MITATT DIODES}

The MITATT oscillator is a device which uses both tunneling and impact ionization effects to generate carriers. This makes the device a cross between an IMPATT and TUNNETT device. Elta, et al. have reported a $150 \mathrm{GHz}$ MITATT which when operated in CW mixde produced abo'st 3 milliwatts of power [ref 43]. Like TUNNETT's these devices should be able to operate with reasonable efficiencies in the range of 100 to $800 \mathrm{~Hz}$.

\subsection{DOVETT DIODES}

Although there are no experimental results from DOVETT (double velocity transit time) devices, efficiencies in excess of $25 \%$ have been predicted [ref 28]. DOVETT s are related to BARITTs except the velocity of carriers near the injection contact is significantly less than near the collection contact (in the DOVETT device). The negative resistance of a DOVETT can be very high and can operate without a 
barrier injection delay. This allows the device to operate at a higher DC bias than BARITTs. These advantages give DOVETT's a higher efficiency and probably a capability to operate well into the millimeter range of frequencies.

\subsection{FET'S AND BIPOLAR TRANSISTORS}

Field effect transistors have been shown to operate up to $110 \mathrm{GHz}$ [ref 44]. Although power devices are not available which are capable of producing millimeter waves, FETs are especially useful for low noise amplifiers and millimeter wave integrated circuits for direct signal processing. FET oscillators have operated at $12 \%$ efficiency at $35 \mathrm{GHz}$ which compares favorable with the efficiency of IMPATT devices at the same efficiency [ref 44].

The most common FET structure for millimeter wave applications is the MESFET (metal semiconductor FET). A recent development is the MODFET (modulation doped FET) which promises to improve the noise figure of FETs above that of MESFET's [ref 45]. Currently there are no results for MODFETs in the millimeter band.

Bipolar transistors, while theoretically capable of operating in the millimeter band, suffer from serious consideration because of the many advantages that FET devices have over bipolar's including: higher voltage and current gain, higher efficiency, lower noise figure, higher operating frequencies, and higher input impedance.

\subsection{HARMONIC GENERATORS}

Solid state diodes have been used in cross guide configurations to achieve frequency multiplication. Multipliers are usually two por devices in which input power enters one port and frequency multiplied power exits the other port. These multipliers are capable of high efficiency operation (15\% to 35\%) at powers approaching 100 milliwatts at $180 \mathrm{GHz}$ by using carefully tuned geometries to suppress harmonics. Because these multipliers are tuned so carefully, the frequency cannot be varied over more than about one tenth of one percent. Useful power has been obtained from these multipliers up to about $600 \mathrm{GHz}$ [ref 27]. 


\subsection{POWER COMBINING}

Because solid state devices have many advantages over vacuum electronic devices except for power capability it would be advantageous to combine the power of several solid state sources. There are two basic methods for power combining: circuit combining and device combining.

Circuits (oscillators or amplifiers) can be combined in three ways: N- way combining, tree or chain combining, and hybrid approaches. $\mathrm{N}$-way devices are combiners which combine the power of several devices (oscillators or amplifiers) in a cavity or nonresonant structure. The diodes lock together due to intermode coupling provided by the resonator (see Figure 4-15). These combiners can have efficiencies which exceed $90 \%$ at frequencies up to $300 \mathrm{GHz}$. Examples of tree and chain structures are shown in Figure 4-16. These structures require several stages of combining and are therefore not as efficient as $\mathrm{N}$-way combiners. Hybrid combiners can be used with a small number of devices. Currently, about four diodes can be combined at $140 \mathrm{GHz}$.

The device combining technique combines semiconductor devices at the chip level. The devices are clustered in an area which is small compared to the wavelength of the generated wave. Stabilizing capacitors must be used in many of the chip combiners adding somewhat to the complexity. Efficiency of $82 \%$ has been achieved at $40 \mathrm{GHz}$ using a multichip combiner. Further work in this area needs to be done before its full potential can be evaluated.

\subsection{MANUFACTURERS [REF 46]}

Millimeter wave silicon, GaAs IMPATT, and Gunn devices are available from Hughes, Raytheon, and Varian. Hughes has a complete catalog of silicon IMPATT diodes up to frequencies of $140 \mathrm{GHz}$. NEC and Thomson CSF have commercial devices up to $94 \mathrm{GHz}$. GaAs IMPATT diodes are not available because of reproducibility concerns.

GaAs Gunn devices are available commercially from Varian, Central Microwave, M/A Com, Hughes, Alpha-TRG, and Plessey. High power InP Gunn devices are not commercially available although Varian will make them on a special basis. 


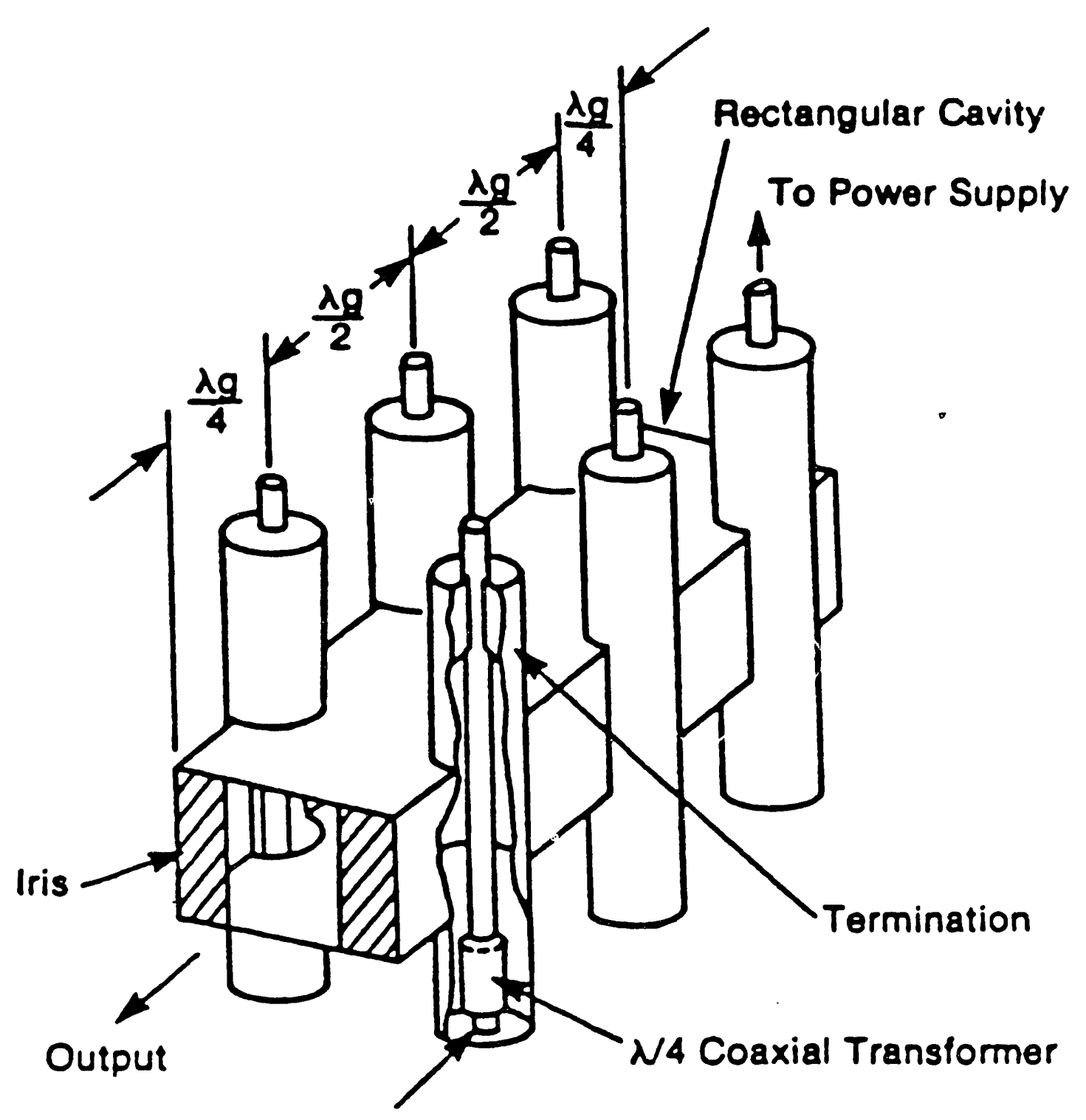

II

Diode

Figure 4-15. Power Combining Using Rectang:lar Resonator (Reference 31).

] 


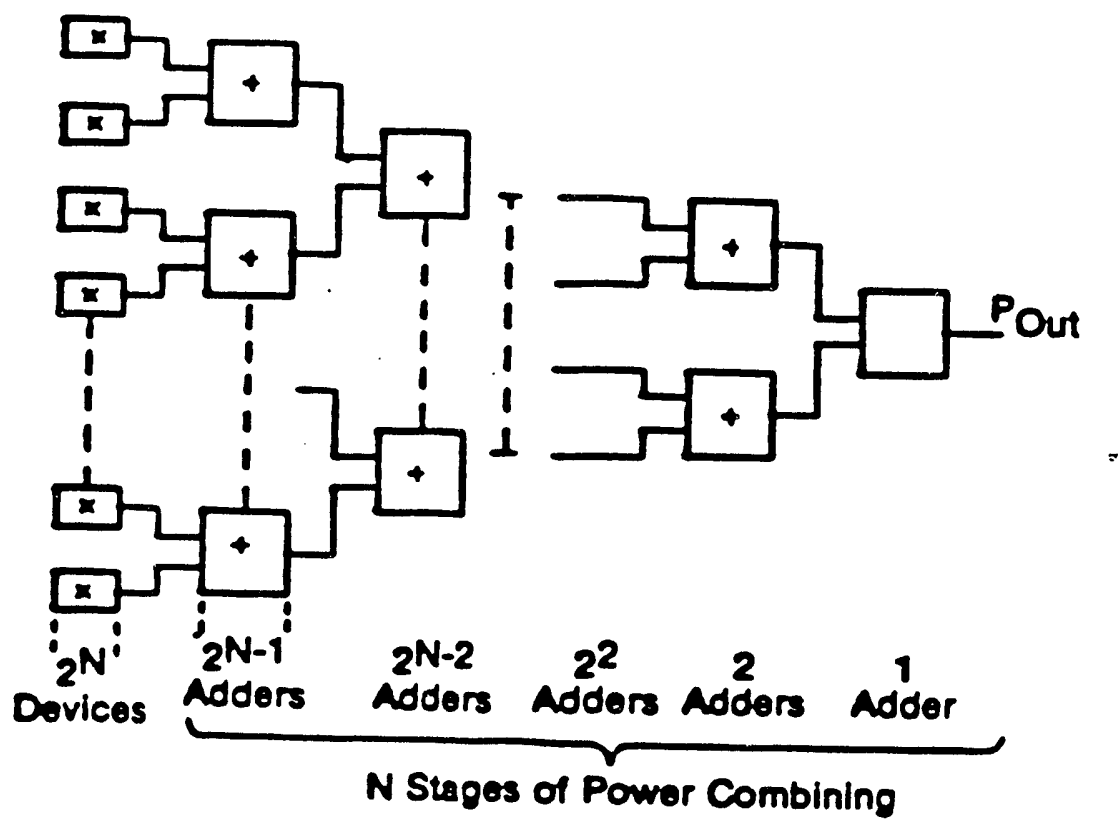

(a)

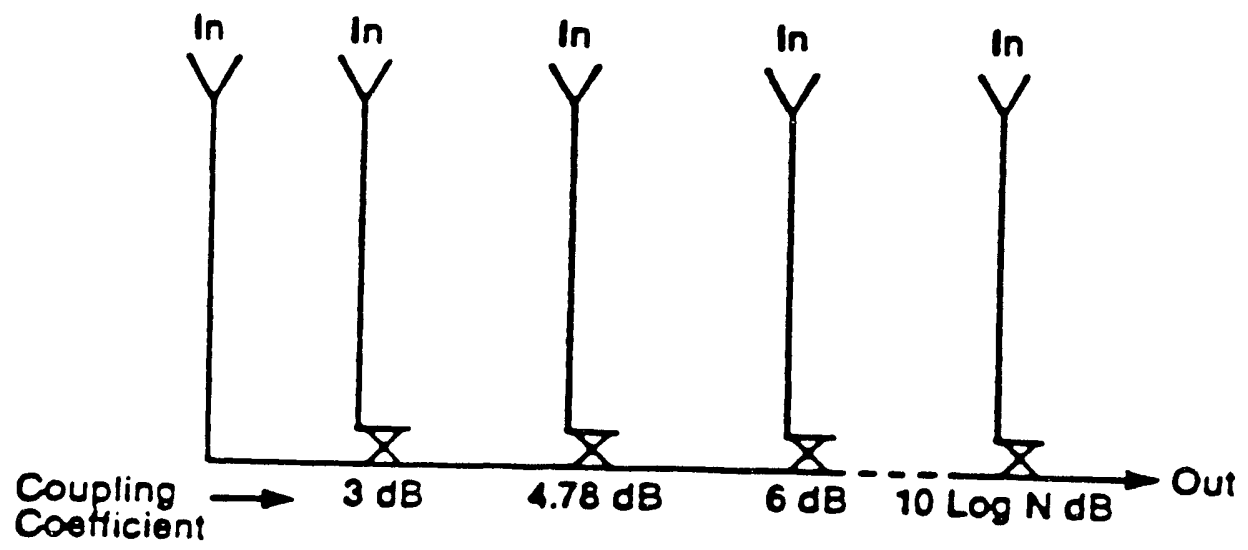

(b)

Figure 4-16. Power Combining Configurations. (a) A tree power combining network; (b) a chain combining structure. (Reference 31) 
Hughes is the major supplier of millimeter wave transmitter and receiver components. Alpha-TRG is a major supplier of receiver components.

1

!)

]

1

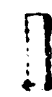

1

11

I

!

1

I]

I)

4-28




\section{SECTION 5 \\ VACUUM ELECTRONICS SPECIFICS}

This section presents a short summary of vacuum electronic devices that supplements the overview of that topic in Section 2.2. To begin with, it is useful to broadly categorize the various devices in so that their characteristics and potential, particularly the tunability, can be understood. First, a most basic technical point concerns electron-photon energy exchange. To transfer energy from the e-beam to the photon EM mode, matching is required between the EM phase velocity and the velocity of the space charge bunches of the e-beam. Since the e-beam moves with less than the velocity of light, either the optical wave must be slowed or the effective speed of the e-beam increased. Devices in which the electromagnetic phase velocity is slowed are called slow wave devices, and the others are fast wave devices.

\subsection{CONVENTIONAL SLOW WAVE DEVICES}

The hear of the slow wave device is the slow wave structure. Several examples are shown in Figure 5-1 along with the names of the devices in which they are typically found. In these conventional, mature devices, the structure is fabricated of metal and has transverse dimensions comparable to the free space wavelength. It can be thought of as a waveguide. If the internal surface of the waveguide were smooth, then the phase velocity would always exceed the velocity of light, rendering it useless for exchanging electron and photon energy. However, by loading the waveguide with periodic disruptions, the phase velocity can be adjusted to values less than the light velocity.

If the disruptions are small, they have little effect except when their spacing is chosen so that the interferences add in phase. This leads to a dispersion diagram that contains regions of pass bands, in which a wave propagates, and stop bands, in which the tube presents a reactive load and a will not transmit energy. As the size of the disruptions increase, the pass bands become narrow and the stop bands are wide. Depending on the details, a particular structure might support a standing wave or a travelling wave, and in the latter case the phase and group velocities could be in the same or opposite directions. 
a. Circular geometry of magnetron

!)

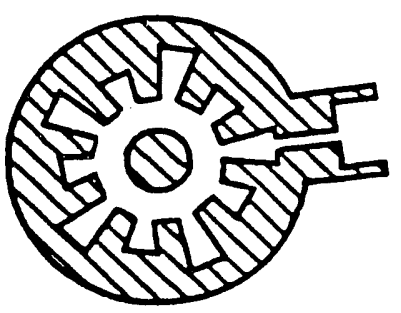

b. Standing wave cavities of klystron

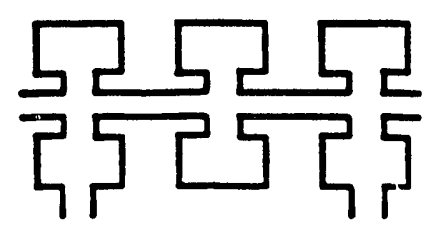

c. Helix of travelling wave tube (TWT)

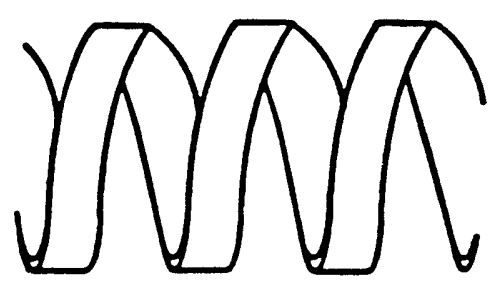

d. Vane geometry of backward wave oscillator (BWO)

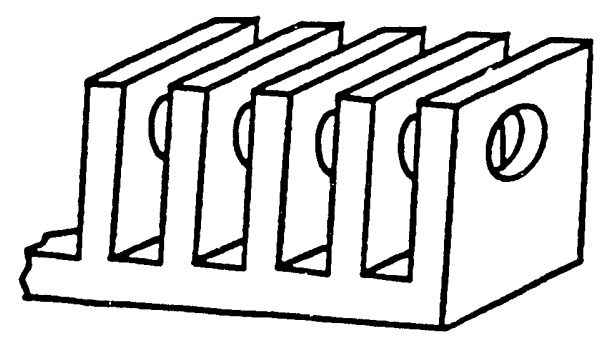

9019096

Figure 5-1. Examples of Slow Wave Structures (taken in part from Reference 6). 
Several slow wave structures are displayed in the Figure 5-1. The magnetron and klystron are examples of standing wave devices. Their pass bands are quite narrow, their tunability is low unless mechanical adjustment is made, and their machining tolerances are severe at short wavelengths. By comparison, the structure of the travelling wave tube (TWT) and backward wave oscillator (BWO) have superior dispersion characteristics which lead to broad band operation, approaching one octave. In the BWO the group velocity is directed opposite to the phase velocity, so that the output energy is taken from the cathode end.

\subsection{WIDE APERTURE SLOW WAVE DEVICES}

The slow wave sources discussed above share the common characteristic that their transverse dimensions are less than the free space wavelength of the radiation they produce. This leads to poor scaling to millimeter wavelengths along with increasingly limited power capability due to the need for adequate heat dissipation. Some aspects of this difficulty can be overcome with large aperture slow wave structures. Two examples are shown in Figure 5-2, the Ledatron [ref 6] which uses an open grating concept, and the Cherenkov laser [ref 7], which uses a dielectric-loaded smooth waveguide. Both are laboratory items at present with no commercial equivalent.

While there are two modes of operation for the ledatron, the simplest and most tunable involves an interaction not unlike that of an extended interaction klystron, but the klystron cavity mode is replaced with the standing wave in a simple Fabry-Perot resonator that operates single mode by virtue of the mirror shapes and spacing. The mode is chosen so that the E-field is perpendicular to the grating groove lines. This means that near the grating where the e-beam will pass, the E-field is small immediately above the groove peaks, but is large (and oscillating) immediately above the groove troughs. A sheet electron beam directed over the top of the grating can then exchange energy with the standing wave. One view of this interaction is that the grating is shielding the electrons from the E-field at time when the phase is not favorable, and exposing the electrons to the field when the phase is favorable.

A tuning range of only about $1 / 2$ percent is possible with beam voltage alone, but this can be extended to 40 percent if the spacing between the flat mirror and grating 

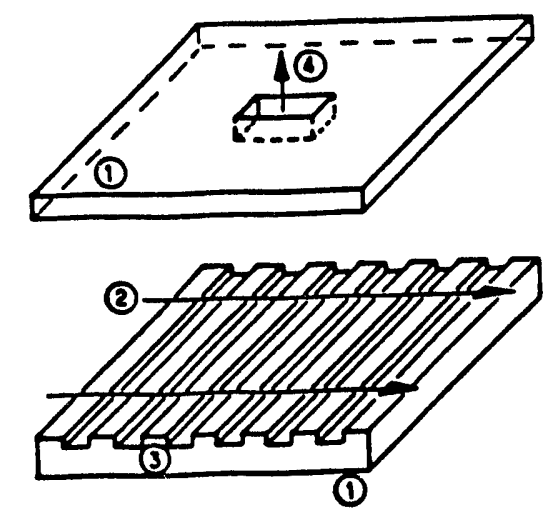

II

a) Open Grating Resonator of Leadatron.
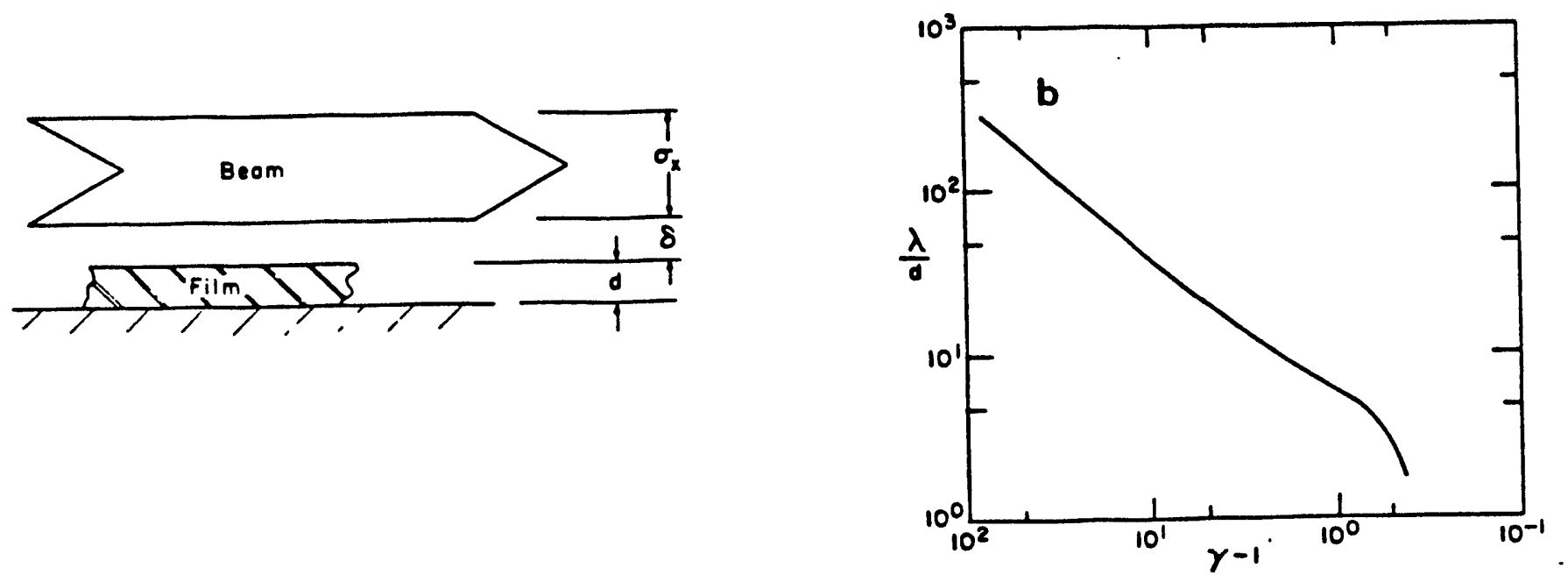

b) Dielectric - Guided Surface Wave of Cherenkov Laser and Theoretical Tuning with Variation of E-beam Energy $\gamma$.

Figure 5-2. Overmoded, Wide Aperture Slow Wave Structures (taken from References 7 and 10). 
is also adjusted. While the system has the fabrication advantage of a large cavity, there is still a drawback regarding heating in that the e-beam must have close proximity to the grating surface.

The geometry shown in Figure 5-2.b allows a Cherenkov interaction between a sheet electron beam and a slow TM mode, guided by a thin dielectric film on a metal sheet. Cherenkov emission is possible whenever the e-beam velocity matches or exceeds the light velocity in the dielectric. The emitted wavelength will be that for which the electron velocity matches the phase velocity of the guided wave. That this condition should exist at all can be understood as follows. When $\lambda / d>1$, the EM wave is mostly outside the dielectric and it therefore has phase velocity approaching $\mathrm{c}$, while at short wavelengths the wave has significant energy in the film and the phase velocity approaches $\mathrm{c} / \mathrm{n}$, where $\mathrm{n}$ is the dielectric constant. There will be some intermediate wavelength, roughly comparable to the thickness of the dielectric, that will have a phase velocity between $\mathrm{c}$ and $\mathrm{c} / \mathrm{n}$ that can match with e-beams of roughly 1 MeV energy.

A wide tuning range is possible by variation of the e-beam energy, and an example of a calculated tuning curve is included in the figure. Although this calculation covers two orders of magnitude in wavelength, good coupling between the e-beam and guided wave provides sufficient gain only over a much narrower, but still impressive range. The restriction results in part from the inability to optimally match the e-beam size to the optical mode over a wide frequency range.

To date the experiments of Walsh [ref $7,20,21]$ have produced an approximate three-fold tuning range for a single device, spanning 375 to $1000 \mu \mathrm{m}$. As with the ledatron, the Cherenkov laser still requires close proximity between the $\mathrm{e}$ beam and a dielectric surface.

\subsection{FAST WAVE DEVICES}

The fast wave devices break away from the need for proximity of the e- beam to a surface, providing a clear path to high power operation by way of improved thermal management. Two examples are the gyrotron and free electron laser (FEL). In both cases the electromagnetic wave moves with phase velocity very nearly $c$, and the orbit of the e-beam is manipulated to achieve phase matching. 
Figure 5-3 (a) shows basics of the gyrotron operation. An electron beam with a high degree of transverse energy is injected into a solenoidal field within a circular waveguide. Electrons undergo interwoven helical trajectories that, when phased cooperatively, can produce azimuthal E- fields at specific radii and thereby couple to predetermined TE cavity modes operating usually at the cyclotron frequency of the applied magnetic field or its first harmonic. The necessary phase bunching occurs as the electrons relativistic mass changes slightly while they exchange energy with the field. An important quality of the gyrotron dispersion properties is that the majority of the transverse energy can be converted into the EM wave before synchronism is no longer possible, permitting system efficiencies routinely in the 20-40 percent range.

While the gyrotron has a geometry amenable to high power, it is not generally tunable even though the frequency is determined by the magnetic field, which in general is highly adjustable. The reason is that e-beam envelope and cavity shape must be carefully chosen so that only one TE mode is driven over threshold, otherwise the efficiency drops dramatically. An example of how severe the competition can be is shown in Figure 5-3 (b). Here the threshold currents are calculated [ref 9] for a specific $245 \mathrm{GHz}$ experiment intended to operate on the 2 nd harmonic of the cyclotron frequency and with TE 051 mode. Note how the TE 1021 and TE 051 modes have nearly identical excitation strengths. For calculations such as this a specific e-beam spatial profile is assumed, and mode competition can be even worse if in practice there are excessive deviations from the proper beam profile. As mentioned in Section 2.2, these problems can in principle be largely suppressed if open, optical style, resonator techniques are employed and their mode selection properties exploited. This is a key growth area for the gyrotron community, and may lead to simultaneous high power, high efficiency, and tunability.

The other fast wave device for special consideration is the free electron laser (FEL). There is no other source which has the same potential for versatility, including tunability, high power capability, and general freedom with respect to temporal pulse shape. Indeed, the FEL is at the same time proposed as a means to destroy mi:isile warheads half a world away, and as a path to new medical advances. Whether it achieves these goals or the many other optimistic predictions of the last decade remains to be seen. 
a)
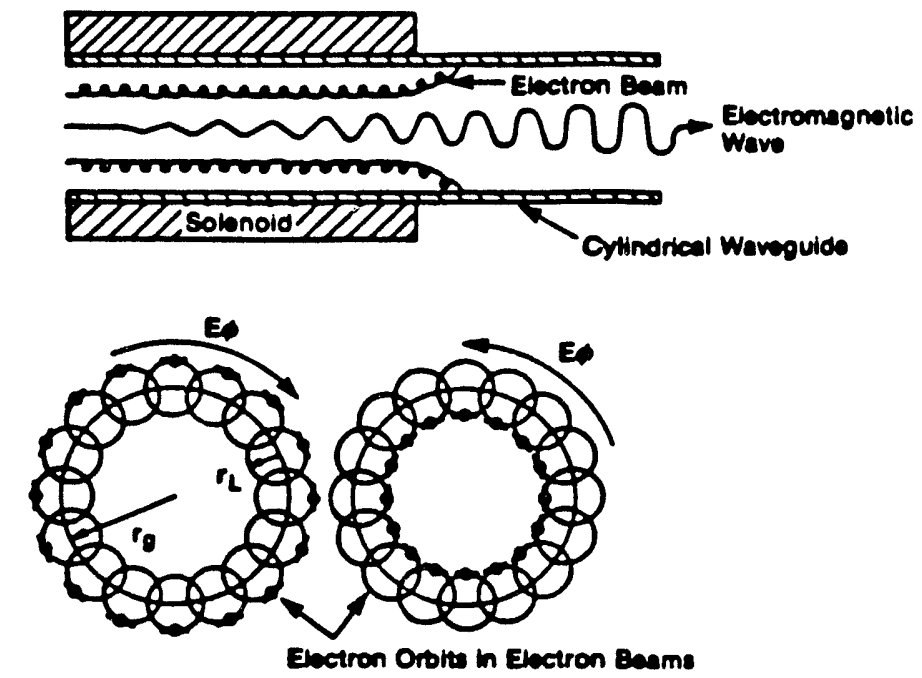

b)

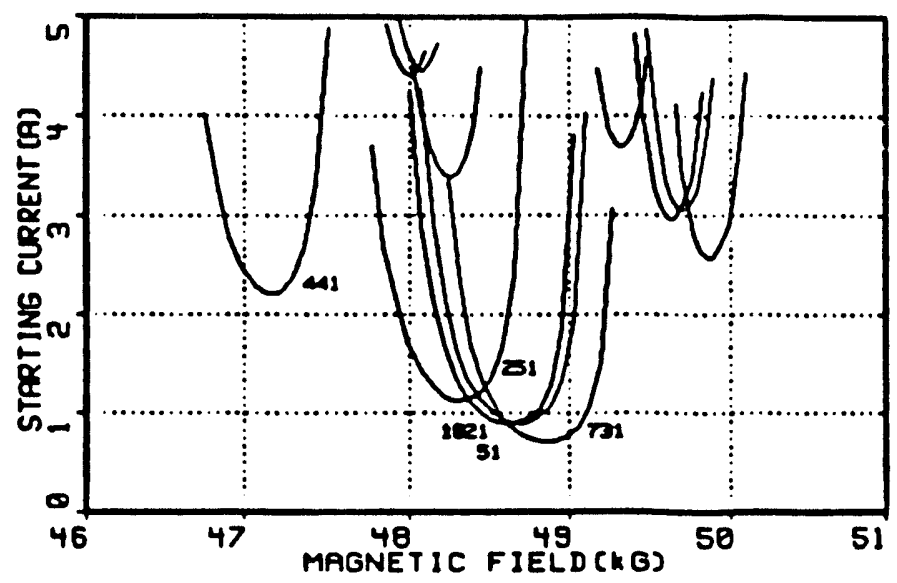

Figure 5-3. Gyrotron. a) basic geometry and electron orbits, b) example of mode completion. Taken from References 6 and 9. 
The general FEL concept is shown in Figure 5-4. The energy exchange between electrons and photons proceeds by means of a synchronism within the wiggler between the transverse component of the electron velocity and the transverse field of the optical wave. The wiggler bore can be large, in fact the entire wiggler can, in principle, be replaced with a low frequency EM wave propagating counter to the e-beam, so there is no fundamental requirement for close proximity of the e-beam to any surface. In reality, FEls with good electron-photon coupling have small wiggler bores to provide the highest possible magnetic field. This is similar to the situation with the gyrotron if the waveguide is replaced with an optical cavity. There will be a tendency to small bores whenever high magnetic fields are required.

In contrast to the conventional waveguided gyrotron, the FEL mode control is accomplished with the end mirrors so proximity to the bore surface is for that reason is unnecessary. Also distinguishing the FEL from the gyrotron is the the lack and any frequency limitation due to magnetic field strength limitation. Synchronism is easily achieved, in various devices, from millimeter to $x$-ray wavelengths. This tunability has been demonstrated in various experimerits over the last several years. The initial Los Alamos device [Ref 17] covered approximately 9 to 35 microns, the Mark IInow being reconstructed at Duke University lases over the 2.0 to 5.5 micron range [ref 11], the LLNL induction linac FEL, ELF [ref 15], has operated at 2 to $8 \mathrm{~mm}$, and the VEPP-3 storage ring FEL at Novosibirsk [ref 16] has covered the range from 240 to $690 \mathrm{~nm}$.

Notwithstanding the potential for versatility, the FEL, with its complex, expensive, and bulky hardware, is not replacing other sources and is not likely do so in the near future except in relatively narrow applications, for example those requiring very high average powers (an as yet unproven capability). There is a broad application area for the FEL in the source poor regions of the IR spectrum.

The FEL has good scaling potential from the IR, where it has been most successful, toward the millimeter and microwave region. The electron- photon coupling strength increases rapidly at the longer wavelengths. This coupling is, in a simplified sense, a measure of how well the electron velocity can be aligned with the E-field of the desired optical mode. While this scaling is favorable, the more traditional millimeter sources have good coupling already with the TM modes of the slow wave structures and the high transverse e-beam velocity of the gyrotron. Thus 


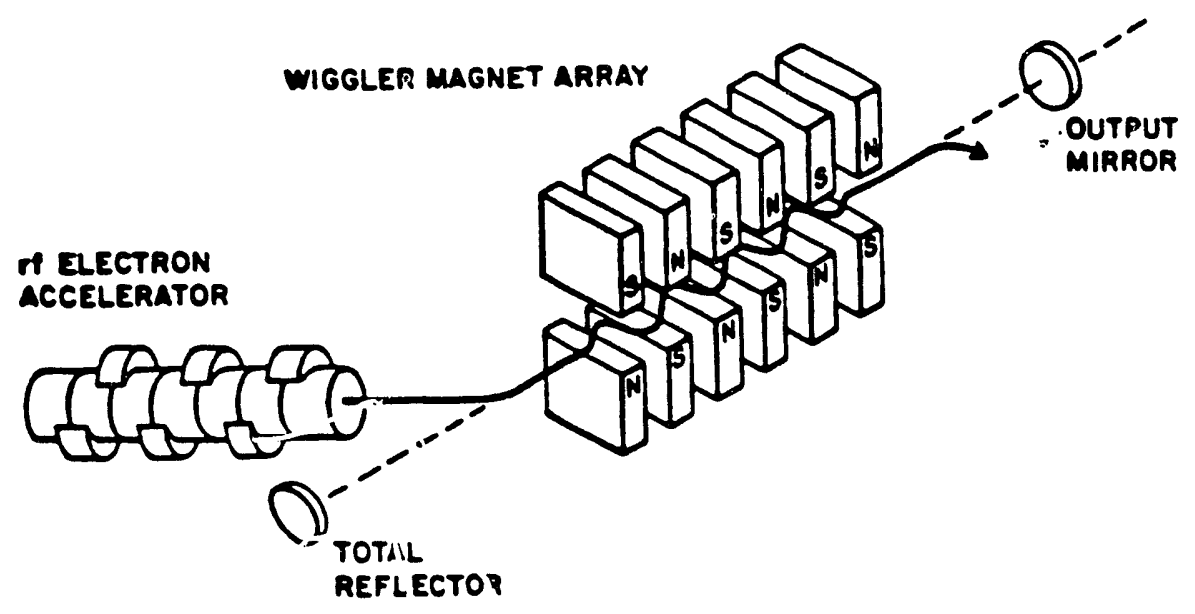

Figure 5-4. FEL Concept (taken from Reference 19). An electron beam passing through the wiggler exchanges energy with a TEM optical wave by means of a small transverse velocity component. 
the fact the the FEL can also have comparable coupling in millimeter region is actually a way to see that its coupling becomes quite poor at the shorter wavelengths. In fact, establishing laser action at all in the IR and especially the visible can be challenging.

If tunability were ignored, it is not likely that the FEL would compete well with the gyrotron up to the maximum reasonable gyrotron frequency of a few hundred GHz.

Altinougin the FEL electron-photon coupling scales well to millimeter wavelengths, these longer wavelengths tend to favor lower e-beam voltages and that in turn leads to beam control problems in wide aperture systems. An axial magnetic guide field is often necessary as the beam voltage drops below several MeV, which is typically the case in millimeter systems. This applies to typical experiments to date involving kilo-ampere currents and higher [ref 49]. The millimeter FEL can be made to work at much lower currents, and with much improved electron transport, if the optical gain and laser efficiency are reduced, but this regime is yet to be explored. The interaction between the guide and wiggler fields complicates the orbits in a generally deleterious manner [ref 50] compared to what would be possible if somehow the space charge problem could be circumvented without the guide field.

While FEL resinance can be achieved at virtually any frequency, the optical system will limit the usable tunability. Optical parameters which must be controlled over the full bandwidth of operation include, longitudinal and transverse mode selection, mode size, cavity losses, and cavity outcoupling. As yet there has been little development for highly broadband cavities, mostly because designs to date have been highly optimized to achieve maximum performance. After excellent narrow-band performance is achieved, the broadband development work will start.

One positive aspect of this problem for the FEL at the longer wavelengths (millimeter and microwave) is that the optical gain can be so large that the cavity need not operate near optimum. In the IR and visible the need for near-optimum systems compromises the opportunity for broad banding. One octaves tuning range may be the reasonable limit for good power and efficiency in the $\mathbb{R}$, with perhaps two octaves in the millimeter and microwave range. In any event the FEL is not likely to be a compact, simple source any time soon. 


\section{SECTION 6 \\ SUMMARY AND CONCLUSIONS}

This report has summarized the nature, capabilities, and potential of existing millimeter and microwave sources in order to provide insight as to the merit of the newly proposed plasma upshift source. In some sense the existing sources are competitors, and it is against that backdrop that the opportunities for new sources must be viewed. Capability for tunability at moderately high powers has been emphasized. At low powers, on the order of watts, the development of the IMPATT diode has filled many of todays application needs. At the higher powers there exists a broad array of devices; a mature group with little broad band capability, and new devices which do have potential for broadbanding.

The capabilities of the vacuum electronic sources has been discussed in terms of the development paths and the underlying characteristics that lead to various degrees of versatility. It has been intended that this will enable the reader to gain a perspective on what potential properties are required of new concepts if they are to be of interest. A clear trend has been identified in the sources being now developed in that they utilize broadband resonance mechanisms with a minimum of mechanical precision required. The plasma upshift source fits in this progression along with the gyrotron, ledatron, Cherenkov lasers, and the FEL. With several broadband mechanisms now demonstrated, the development challenge is to combine them with broadband optical systems.

The application potential for the plasma upshift source looks particularly bright in the area of time-dependent frequency shifting, particularly concerning shifts which occur on a period by period basis. This specific case is of high interest today in the area known as impulse radar, or crafted wave radar. The plasma upshift source may be the appropriate driver for a wide band power amplifier, such as the gyrotron. The advantages of ultra wide band radar are not yet proven, but should they becomes so, funding for crafted wave sources will skyrocket. Independent of the radar, the plasma upshift source has potential above $100 \mathrm{GHz}$, the point at which inexpensive sources are as yet unavailable. 


\section{REFERENCES}

1. S.C. Wilkes, J.M. Dawson, and W.B. Mori, "Frequency Up-Conversion of Electromagentic Radiation with Use of an Overdense Plasma," Phy. Rev. Lett. Vol. 61, 337 (1988).

2. C. Joshi, C.E. Clayton, and K. Marsh, "Demonstration of Frequency Upshifting of Microwave Radiation by Rapid Plasma Creation," to be published in app. Phys. Lett.

3. "Ultra Wideband Radar Research and Development," report prepared for Naval Ocean Systems Center, San Diego, California by the Mechanical and Electronic Engineering Division of Los Alamos National Laboratory, June 1989.

4. "Impulse Radar Should Be Explored," Aviation Week and space Tech. pg. 9, February 26, 1990.

D.F. Bond, "USAF Believes Impulse Radar Not Feasible for Detecting B-2," Aviation Weekend Space Technology, pg. 53, February 26, 1990.

5. R.K. Parker and R.H. Abrams, "Radio-Frequency Vacuum Electronics: A Resurgent Technology for Tomorrow!," SPIE Millimeter Wave Technology IV and Radio Frequency Power Sources, Vol. 791, 2 (1987).

6. P. Bhartia and I.J. Bahl, Millimeter Wave Engincering Applications, John Wiley \& Sons, New York, 1984.

7. E.P. Garate, J. Walsh, C. Shaughnessy, B. Johnson, and S. Moustaizis, "Cherenkov Free-Electron Laser Operation From 375 to 1000 Microns," Nucl. Instr. and Meth. in Phys. A259, 125 (1987).

8. A.L. Throop, Nucl. Inst. and Meth. A272, 15 (1988).

9. K.J. Button, editor, "Infrared and Millimeter Waves," Coherent Sources and Applications, Vol. 7, Part II, Academic Press, New York, 1983.

10. K.J. Button, editor, "Infrared and Millimeter Waves," Coherence Sources and Applications, Vol. 5, Part I, Academic Press, New York, 1982. 
11. S.V. Benson, J. Schultz, B.A. Hooper, R. Crane, and J.M.J. Madey, Status Report on the Stanford Mark III Infrared Free-Electron Laser, pg. 22 in "FreeElectron Lasers," edited by P. Sprangle, C.M. Tang, J. Walsh. Published by North Holland, Amsterdam, 1988.

12. T.S. Phalen, S.B. Hutchinson, and T. McNulty, "Optical Beam Integration System," United States Patent No. 4,733,944, Issued 1988.

13. G.R. Thoren, "Advanced Applications and Solid-State Power Sources for Millimeter-Wave Systems," SPIE, Millimeter Wave Technology III, Vol. 544, 2 (1985).

14. G. Bekefi, "Survey of Physics Research in Microwave Devices," IEEE International Electron Devices Meeting, Dec. 1984. Technical Digest, pg. 822 (1984).

15. T.J. Orzechowski et al., Phys. Rev. Lett. Vol. 57, 2172 (1986).

A.L. Throop, et al., "Experimental Results of a High Gain Microwave FEL Operating at $140 \mathrm{GHz}$," Nucl. Instru. and Meth. in Phys. Research, Vol. A272, 15 (1988).

16. V.N. Litvinenko, "Veep-3 Storage Ring Optical Klystron: Lasing in UV," Synchrotron Radiation News, Vol. 1, 18 (1988).

G.N. Kulipanou, "Veep-3 Storage Ring Optical Klystron: Lasing in Visible and Ultraviolet Regions," 11 th International Conference on Free-Electron Lasers, August 1989, Naples, Florida, IEEE Conference Digest, pg. 306, (1989).

17. B.E. Newman, "Extension of Free-Electron Lasers into the Extreme Ultraviolet," IEEE Critical Reviews of Optical Science and Technology, Free-Electron Lasers, Vol. 738, 155 (1987).

18. B.W. Henderson, "Superfast Switch Key to Ultra-Wideband Radar, High Power Microwave Systems," Aviation Week and Space Technology, pg. 55, February 26, 1990.

19. C.A. Brau, Free-Electron Lasers, Academic Press, Boston, 1990. 
20. J.E. Walsh, T.C. Marshall, and S.P. Schlesinger, "Generation of Coherent Cherenkov Radiation with an Intense Relativistic Electron Beam," The Phys. of Fluids, Vol. 24, 709 (1977)

22. J.M. Baird, "Survey of Fast Wave Tube Developments," IEEE International Electron Devices Meeting, Tech. Digest, pg. 156, (1975).

23. J. Fienstein, "Status Review of Research on Millimeter Wave Tübes," IEEE Trans. on Electron Devices, Vol. ED-34, 461 (1987).

24. W. Schockley, "Negative Resistance Arising from Transit Time in Semiconductor Diodes," Bell Syst. Tech. J., 33, 799 (1954).

25. R.L. Johnston, B.C. Dehoach, R., and B.G. Cohen, "A Silicon Diode Oscillator," Bell Syst. Tech. J., 44, 369 (1965).

26. C.A. Brackett, "The Elimination of Tuning Induced Burnout and Bias Circuit Oscillation IN IMPATT Oscillators," Bell Syst. Tech. J., 52, 271 (1973).

27. N.C. Currie and C.E. Brown, Principles and Applications of Millimeter-Wave Radar, Artech House, Norwood, MA (1987).

28. S.M. Sze, Physics of Semiconductor Devices, John Wiley \& Sons, New York (1981).

29. H.J. Kuno, "IMPATT Devices for Generation of Millimeter Waves," Chapter 2 in Infrared and Millimeter Waves, Vol. 1, K.J. Button, Academic Press, New York (1979).

30. N.B. Kramer, "Sources of Millimeter-Wave Radiation: Traveling-Wave Tube and Solid State Sources," Chapter 4 in Infrared and Millimeter Waves, Vol. 4, K.J. Button and J.C. Wiltse, New York, Academic Press (1981).

31. P. Bhartia and I.J. Bahl, Millimeter Wave Engineering and Applications, John Wiley \&Sons, New York (1984).

32. J.B. Gunn, "Microwave Oscillation of Current in III-V Semiconductors," Solid State Commun., 1, 88 (1963).

33. L. Esaki, "New Phenomenon in Narrow p-n Junctions," Phys. Rev., 109, 603 (1958) 
34. O.P. Handhi, Microwave Engineering and Applications, Pergamon Press, New York (1981).

35. C.A. Burrus, "Millimeter Wave Esaki Diode Oscillator," Proc. IRE, 48, 202 (1960).

36. M.E. Elta and G.I. Haddad, "High Frequency Limitations of IMPATT, MITATT, and TUNNETT Mode Devices," IEEE Trans. Microwave Theory Tech., MTT-27, 1029 (1978).

37. D.S. Pan and N. Lee, "GaAs Abrupt Junction MITTATT and TUNNETT," 6th International Conference on Infrared and Millimeter Waves, Miami Beach, FL, paper M-5-3 (1981).

38. J.I. Nishizawa, K. Motoya, and Y. Okuna, "GaAs TUNNETT Diodes," IEEE Tans. Microwave Theory Tech., MTT-26, 1029 (1978).

39. J. Nishizawa, K. Motoya, and Y. Okuno, "Submillimeter Wave Oscillation From GaAs TUNNETT Diode," 9th European Microwave Conference, Brighton, UK (1979).

40. D.J. Coleman, Jr. and S.M. Sze, "The BARITT Diode - A New Low Noise Microwave Oscillator," IEEE Device Res. Conf., Ann Arbor, Mich. (1971).

41. P.N. Forg and J. Feyer, "BARITT Diodes Advance to Ka Band," Microwaves, $\underline{20}$, 61 (April 1981).

42. H.J. Prager, K.K.N. Chang, and S. Weisbroad, "High Power, High Efficiency Silicon Avalanche Diodes at Ultra High Frequencies," Proc. IEEE, 55, 588, (1967).

43. M.E. Elta, H.R. Fetterman, W.V. Macropoulous, and J.J. Lambert, "150 GHz GaAs MTTATT Source," IEEE Electron Device Letters, EDL-1, 115 (1980).

44. H.Q. Tserng and B. Kim, "110 GHz GaAs FET Oscillator," Electronics Letters, 21. 178 (1985).

45. R.Fischer and H. Morkov, "New High Speed (A1GaAs) Modulation Doped Field Effect Transmitter," IEEE Circuits and Devices Magazine, 1, 35 (July 1985). 
46. R.s. Ying, "Solid State Sources Power Millimeter Transmitters," Microwave Systems News, 13, no. 12, 280 (1983).

47. R.K. Parker and R.H. Abrams, "Radio-Frequency (RF) Vacuum Electronics: A Resurgent Technology for Tomorrow," Millimeter Wave Technology IV and Radio Frequency Power Sources, SPIE Vol. 791, 2 (1987).

48. F.A. Meyers, "Performance Parameters Examined for Millimeter-Wave Solid State Devices," Microwave Systems News, 14, no. 7, 266 (1984).

49. J.A. Pasour, "Powerful Millimeter-Wave FELs Driven by High-Current, MeV Accelerators," SPIE Critical Reviews of Optical Science and Technology, FreeElectron Lasers, Vol 738, 55 (1987).

50. J.A. Pasour and S.H. Gold, "Free-Electron Laser Experiments With and Without a Magnetic Guide Field: A Review of Millimeter Wave Free-Electron Laser Research at the Naval Research Laboratory," IEEE J. Quant. Elect. Vol. QE-21, 845 (1985). 
V. Publications. 


\section{Publications}

1. C. Joshi et al., "Plasma Wave Wigglers for Free-Electron Lasers," IEEE Journal of Quantum Electronics, QE-23, 1571-1578 (1987).

2. C. Joshi et al., "Plasma Wave Wigglers for Free-Electron Lasers," Proceedings of the Particle Accelerator Conference, IEEE Catalog no 87 CH2387-9, p. 199-201 (1987).

3. C. Joshi et al., "Test Particle Injection in the Relativistic Plasma Waves Excited by Two Co-Propagating Laser Beams," Proceedings of the SPIE Symposium of OE LASE '88, January 10-17, 1988, Anaheim, CA

4. C. Joshi, C. E. Clayton, T. Katsouleas, W. B. Mori, "Interaction of Relativistic Electrons with Space Charge Waves Driven by Laser Beams," in Laser Interaction and Related Plasma Phenomena," Vol. 8, ed. by H. Hora and G. Miley, (Plenum Press, 1988).

5. S. C. Wilks, J. M. Dawson, and W. B. Mori, "Frequency Upconversion of E.M. Radiation Using an Overdense Plasma," 61, 3s̄7 (1988).

6. S. C. Wilks, J. M. Dawson, W. B. Mori, C. Joshi, and J. M. Dawson, "A Photon Accelerator," Phys. Rev. Lett. 62, 2600 (1989).

7. Y. Kitagawa, R. L. Savage, Jr. and C. Joshi, "Demonstration of Collisionally Enhanced Degenerate Four Wave Mixing in a Plasma," Phys. Rev. Lett. 62, 151 (1989).

8. R. Williams, C. E. Clayton, C. Joshi, T. Katsouleas, and W. B. Mori, "Theory and Experiments on the Generation of Spontaneous Emission Using a Plasma 
VI. Appendix.

(remome ard eyeled Reparately) 

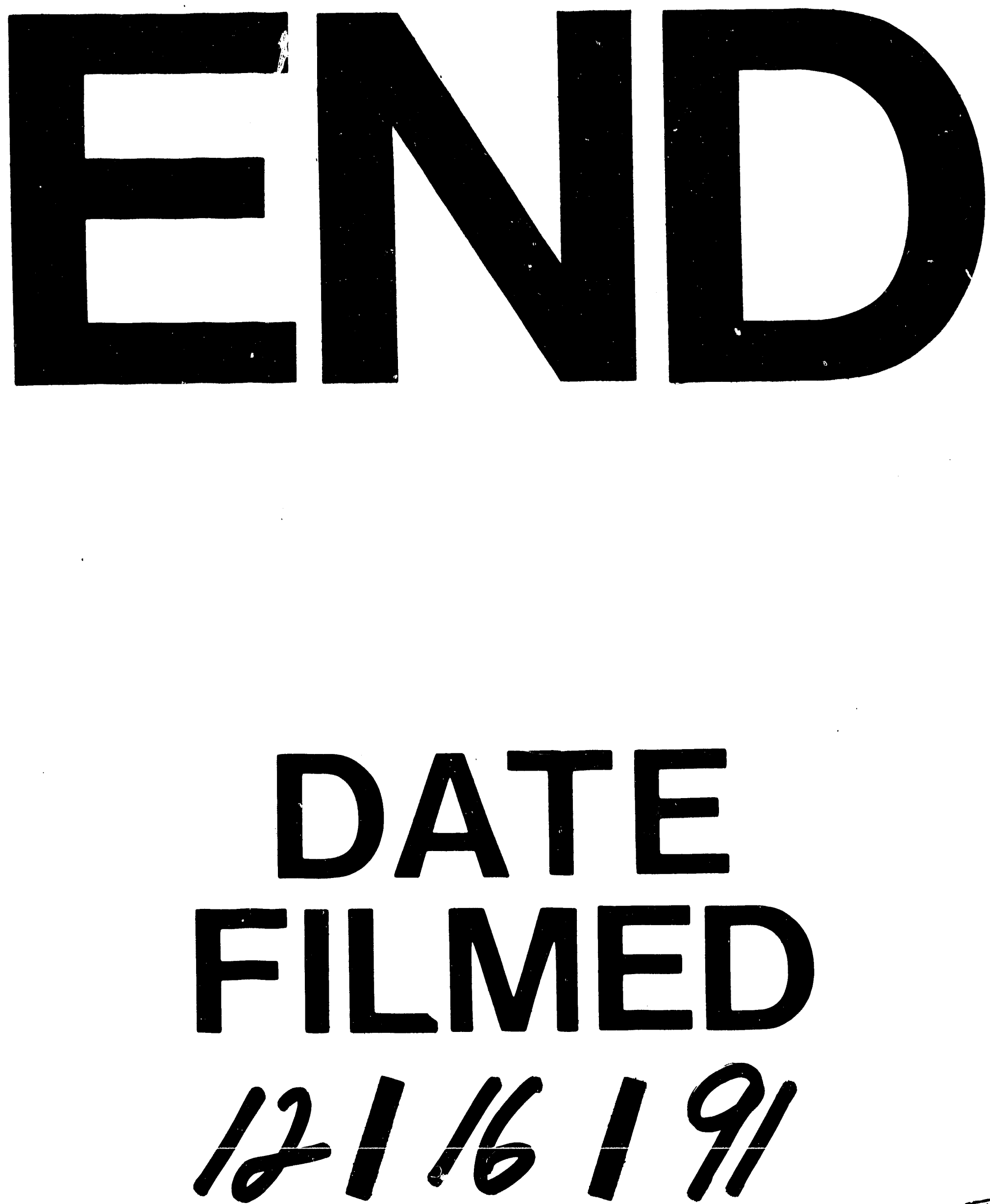



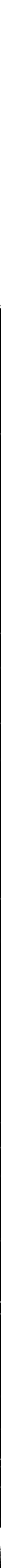

